\title{
Targeting ATCL to rescue BSCL2 lipodystrophy and its associated cardiomyopathy
}

\author{
Hongyi Zhou, ${ }^{1}$ Xinnuo Lei, ${ }^{1}$ Yun Yan, ${ }^{1}$ Todd Lydic, ${ }^{2}$ Jie Li, ${ }^{3}$ Neal L. Weintraub, ${ }^{3}$ Huabo Su, ${ }^{3}$ \\ and Weiqin Chen' \\ 'Department of Physiology, Medical College of Georgia at Augusta University, Augusta, Georgia, USA. ${ }^{2}$ Department of \\ Physiology, Michigan State University, East Lansing, Michigan, USA. ${ }^{3}$ Vascular Biology Center, Medical College of Ceorgia \\ at Augusta University, Augusta, Georgia, USA.
}

\begin{abstract}
Mutations in the BSCL2 gene underlie human type 2 Berardinelli-Seip congenital lipodystrophy (BSCL2) disease. Global Bscl2-- mice recapitulate human BSCL2 lipodystrophy and results in the development of insulin resistance and hypertrophic cardiomyopathy. The pathological mechanisms underlying the development of lipodystrophy and cardiomyopathy in BSCL2 are controversial. Here we report that $B s c / 2^{-/-}$mice develop cardiac hypertrophy because of increased basal IGF1 receptor-mediated (ICF1R-mediated) PI3K/AKT signaling. Bsc/2-/- hearts exhibited increased adipose triglyceride lipase (ATCL) protein stability and expression causing drastic reduction of glycerolipids. Excessive fatty acid oxidation was overt in Bscl $2^{-/-}$hearts, partially attributing to the hyperacetylation of cardiac mitochondrial proteins. Intriguingly, pharmacological inhibition or genetic inactivation of ATCL could rescue adipocyte differentiation and lipodystrophy in Bscl2-cells and mice. Restoring a small portion of fat mass by ATCL partial deletion in $B s c / 2^{-/-}$mice not only reversed the systemic insulin resistance, but also ameliorated cardiac protein hyperacetylation, normalized cardiac substrate metabolism, and improved contractile function. Collectively, our study uncovers pathways underlying lipodystrophy-induced cardiac hypertrophy and metabolic remodeling and pinpoints ATCL as a downstream target of BSCL2 in regulating the development of lipodystrophy and its associated cardiomyopathy.
\end{abstract}

Conflict of interest: The authors have declared that no conflict of interest exists.

Copyright: (c) 2019, American Society for Clinical Investigation.

Submitted: April 23, 2019

Accepted: June 5, 2019

Published: July 25, 2019.

Reference information: /CI Insight. 2019;4(14):e129781. https://doi. org/10.1172/ji.insight.129781.

\section{Introduction}

Adipose tissue dysfunction, which occurs in both obesity and lipodystrophy, is highly associated with insulin resistance and exerts deleterious effects on the heart. Insulin resistance alters the systemic and neurohumoral milieu, leading to perturbations in mitochondrial energy metabolism in the heart that contribute to myocardial dysfunction. Both lipotoxicity and glucotoxicity have been attributed to cardiac hypertrophy and dysfunction in insulin-resistant obesity $(1-3)$ and lipodystrophy $(4,5)$. Fatty acid oxidation (FAO) is the predominant and critical energy source for the adult heart (6). In obesity and type 2 diabetes mellitus, insulin resistance and increased plasma nonesterified fatty acid (NEFA) content chronically raise FAO in the heart, which reduces cardiac efficiency and compromises cardiac function (7). Endogenous myocardial triglyceride (TG) per se has been shown to be not harmful to the heart $(8,9)$, but it is an important contributor to cardiac FAO $(10,11)$. Especially, adipose triglyceride lipase-mediated (ATGL-mediated) intracellular TG hydrolysis plays a central role for cardiac FAO and function in both the healthy and the diseased heart (10, 12). Meanwhile, mitochondrial acetylation, a novel posttranslational pathway regulating the activity of the enzymes involved in FAO and mitochondrial respiration (13), has been positively associated with excessive cardiac FAO in obesity $(14,15)$. Despite intensive studies, efforts are still needed to elucidate the link between adipose tissue dysfunction, insulin resistance, intramyocellular TG turnover, cardiac substrate metabolism, and heart performance in both obesity and lipodystrophy.

Berardinelli-Seip congenital lipodystrophy (BSCL) is a rare autosomal recessive disorder characterized by a near total absence of body fat from birth or infancy. Patients with BSCL develop metabolic abnormalities similar to obesity, including hyperinsulinemia, insulin resistance, and type 2 diabetes mellitus $(16,17)$. They also develop progressive hypertrophic cardiomyopathy, highlighted by concentric left ventricular (LV) hypertrophy 
that ultimately results in ventricular dysfunction and cardiac failure (18-21). Type 2 BSCL (BSCL2), caused by mutations in a gene called BSCL2 (also called Seipin), is the most severe form of BSCL (22). Global Bscl2knockout $\left(B s c l 2^{--}\right)$mice recapitulate human BSCL2 with lipodystrophy, organomegaly, severe insulin resistance, and type 2 diabetes mellitus (23-25) as well as hypertrophic cardiomyopathy, which has been attributed to hyperglycemia and glucotoxicity (4). However, the precise link between adipose tissue dysfunction in BSCL2 lipodystrophy and the development of hypertrophic cardiomyopathy remain understudied.

BSCL2 is an integral ER membrane protein expressed in most tissues, especially adipose tissue (22, 26). In yeast, several functional studies have identified BSCL2 to be involved in regulating lipid droplet (LD) biogenesis and phospholipid metabolism (27-30). Drosophila BSCL2 was shown to directly interact with the sarcoplasmic reticulum/ER $\mathrm{Ca}^{2+}$-ATPase 2 (SERCA2) to regulate ER calcium homeostasis and mitochondrial metabolism $(31,32)$. It was also shown to be required for maturation of nascent LDs by mediating the localization of the TG synthesis enzyme glycerol-3-phosphate acyltransferase 4 (GPAT4) (33). The newly resolved molecular structure of Drosophila BSCL2 argues for a structural and possibly lipid transfer role in organizing LD formation (34). The function of mammalian BSCL2 is still controversial. Human BSCL2 was recently found to assemble as an undecamer and directly bind anionic phospholipids (35). Overexpressed BSCL2-tagged protein interacts with 1-acylglycerol-3-phosphate O-acyltransferase 2 (AGPAT2) (36), LIPIN1 (37), GPAT3 (38), and most recently Promethin (39). However, these data have been mainly generated using non-adipocyte cells overexpressing tagged-BSCL2 protein in vitro, whose relevance in vivo remains unclear. Our laboratory and others have shown that deletion of murine BSCL2 causes unbridled cAMP/PKA-mediated lipolysis, which causes aborted adipocyte differentiation and maintenance $(23,25)$. The molecular function of BSCL2 in regulating lipolysis and adipose tissue biology in vivo still needs to be elucidated.

The present studies were undertaken to identify the mechanisms underlying both BSCL2 lipodystrophy and its associated hypertrophic cardiomyopathy. We first characterized the molecular underpinnings of cardiac hypertrophy and contractile dysfunction in hearts of insulin-resistant $\mathrm{Bscl}^{-1-}$ mice. Given the importance of ATGL in cAMP/PKA-mediated lipolysis, we further tested whether ATGL modulation affects adipose tissue mass and restores cardiac function in BSCL2 lipodystrophy. Our study established the functional significance of ATGL-catalyzed lipolysis in governing adipose tissue mass and cardiac lipid remodeling in BSCL2 lipodystrophy and identified what we believe is a novel posttranslational modification in causing abnormal substrate metabolism and contractile dysfunction in a nonobese diabetic animal model with reduced cardiac steatosis.

\section{Results}

Cardiac hypertrophy in Bscl2 ${ }^{--}$mice is associated with prohypertrophic IGF1R-mediated PI3K/AKT signaling. Hypertrophic cardiomyopathy has been previously reported in $B s c l 2^{-/-}$mice (4), but the pathogenesis of the cardiac phenotype was not systematically characterized. Using our independent line of $B s c l 2^{-1-}$ mice (23), we found that cardiac hypertrophy in $\mathrm{Bscl}^{-/-}$mice was evident as early as postnatal day 10 (P10) and persisted to adult, as evidenced by significantly increased ventricle weight (VW), as well as the elevated ratios of VW to BW and VW to tibia length (TL) compared with age-matched control mice (Supplemental Figure 1, A-C; supplemental material available online with this article; https://doi.org/10.1172/jci. insight.129781DS1). Histological images also showed both increased heart sizes and a thicker ventricular wall (Supplemental Figure 1D). Meanwhile, echocardiography revealed that 3-month-old Bscl2 ${ }^{-1-}$ mice exhibited significantly increased systolic LV wall thickness and chamber diameter with preserved ejection fraction and fractional shortening (Supplemental Figure 1, E-G), indicating the development of compensatory hypertrophy. However, by 6 months of age, $B s c l 2^{-1-}$ mice displayed cardiac dysfunction and decompensation. This was indicated by increased LV chamber diameter, decreased ejection fraction and fractional shortening, and elevated expression of genes associated with pathological cardiac remodeling, such as atrial natriuretic peptide ( $N p p a)$ and brain natriuretic peptide ( $N p p b)$ (Supplemental Figure 1, E-I). Together, these data demonstrate that loss of BSCL2 results in cardiac hypertrophy at early postnatal life and cardiomyopathy during aging.

We next focused on identifying mechanisms underlying cardiac hypertrophy in BSCL2 lipodystrophy. We performed an unbiased RNA sequencing (RNA-seq) analysis in ventricles of 10-week-old mice to investigate early alterations in gene expression of $B s c l 2^{-1-}$ hearts. Among 13,387 genes surveyed, 267 genes were differentially expressed $(0.5<\log 2<-0.5$, adjusted $P$ value $<0.05)$ (Supplemental Table 1$)$. 
Intriguingly, KEGG pathway analyses based on signaling and metabolism identified 4 major KEGG pathways that were significantly altered, i.e., PI3K-AKT (mmu04151), neuroactive-ligand interaction (mmu04080), cytokine-cytokine receptor interaction (mmu04060), and extracellular matrix-receptor (ECM-receptor) interaction (mmu04512) (Figure 1A). A gene signature related to PI3K/AKT signaling was particularly interesting because it is known to be prohypertrophic (40). The main transcriptional fingerprints that were substantially altered in relation to the cardiomyocyte response to PI3K/AKT signaling are shown in Figure 1B. We identified an approximately 2.8-fold upregulation of basal AKT phosphorylation at Ser473 in hearts of $\mathrm{Bscl2}^{-/-}$mice fed ad libitum compared with that of $\mathrm{Bscl}^{+/+}$mice (Figure 1, C and D). Concordantly, the downstream phosphorylation of glycogen synthase kinase $3 \beta$ (GSK3ß) at Ser9 (Figure 1, C and E) and p70S6 kinase (S6K) at Thr389 (Figure 1, C and F) in Bscl2-1 hearts were all elevated by 1.8 -fold and 20 -fold, respectively, suggesting increased protein synthesis. Western blotting using an antibody that indistinguishably detects phosphorylation at Tyr1158/Tyr1162/ Tyr1163 of insulin receptor (IR)/type 1 insulin-like growth factor receptor (IGF1R) detected increased tyrosine phosphorylation of IR/IGF1R in hearts of $\mathrm{Bscl2}^{-/-}$mice fed ad libitum, suggesting heightened upstream insulin/IGF1 signaling (Figure 1G). When IGF1R $\beta$ was specifically pulled down, we observed elevated levels of tyrosine phosphorylation in $B s c l 2^{-/}$hearts (Figure 1H). Plasma IGF1 levels were not significantly increased (Figure 1I), in contrast to severe hyperinsulinemia in lipodystrophic Bscl2 ${ }^{-1-}$ mice (23). Collectively, these data suggest that hyperinsulinemia may trigger IGF1R-mediated activation of $\mathrm{PI} 3 \mathrm{~K} / \mathrm{AKT}$ signaling driving cardiac hypertrophy in lipodystrophic $\mathrm{Bscl2} 2^{-1-}$ mice.

Hearts of lipodystrophic Bscl2 $2^{--}$mice exhibit drastic cardiac lipid remodeling associated with increased ATGL stability and upregulation. Lipodystrophy is associated with ectopic fat deposition (41). To our surprise, quantitative enzymatic analyses identified an approximately $60 \%$ reduction of TG in ventricles of 3-month-old $\mathrm{Bscl2}^{-1-}$ mice, which was further reduced by $80 \%$ in 6-month-old mice compared with $\mathrm{Bscl}^{+/+}$mice (Figure 2A). This was confirmed by electron microscopy, which revealed a near-complete lack of LDs (black arrow) in 6-month-old $\mathrm{Bscl2}^{-/-}$ventricles (Figure 2B). A more comprehensive untargeted lipidomic analysis using ventricles obtained from nonfasting 6-month-old $\mathrm{Bscl2}^{+/+}$and $\mathrm{Bscl2}^{-/-}$ mice demonstrated a tendency toward lower lipid abundance in $B s c l 2^{-/-}(15,911 \pm 1,221)$ versus $B s c l 2^{+/+}$ $(17,499 \pm 2,176)$ mice. Based on the percentage distributions of the 5 broadly classified lipid classes defined by the Lipid MAPS Consortium (www.lipidmaps.org), the $B s c l 2^{-1-}$ hearts were enriched in glycerophospholipids as opposed to $B s c l 2^{+/+}$hearts $(91.9 \%[-/-]$ vs $74.7 \%[+/+], P<0.005)$ but exhibited a greater than 4 -fold reduction of glycerolipids $(5.1 \%[-/-]$ vs $23.4 \%[+/+], P<0.005)$ (Supplemental Figure 2, A and B). The proportions of sphingolipids and sterol lipids were slightly upregulated in $\mathrm{BsCl}^{-/-}$hearts, whereas the proportions of NEFA were relatively comparable between 2 genotypes (Supplemental Figure 2, A and B). A comparison of the total abundances for specific glycerolipids identified an approximate $80 \%, 36 \%$, and $40 \%$ reduction of TG, diacylglyceride (DG), and monoacylglyceride (MG), respectively, in $\mathrm{Bscl}^{-/-}$ventricles (Figure 2C). The total normalized lipid abundances for phosphatidylcholine (PC), phosphatidylethanolamine (PE), phosphatidylinositol (PI), phosphatidylserine (PS), and phosphatidylglycerol (PG) did not differ between the 2 genotypes (Supplemental Figure 2C), whereas the total abundance of phosphatidic acid (PA), a vital lipid that acts as a biosynthetic precursor for the formation of all acylglycerol lipids (42), was significantly lower in the hearts of $B s c l 2^{-/-}$mice than in $\mathrm{Bscl2}^{+/+}$mice (Supplemental Figure 2C). Cardiolipin (CL), the signature phospholipid of mitochondria membranes, showed a tendency of lower abundance in $B s c l 2^{-1-}$ hearts. In particular, the content of tetralinoleoyl (L4)-CL (CL72:08), the dominant CL species in heart (43), was reduced by $87 \%$ in $\mathrm{Bscl2}^{-1-}$ hearts with an obvious shift from L4-CL (18:2) to CL species that contain longer polyunsaturated fatty acids (PUFAs), such as CL76:11-CL(18:1_18:2_18:2_22:6) and CL(84:20)-CL(18:2_22:6_22:6_22:6), etc. (Supplemental Figure 2D). These data suggest a unique lack of cardiac steatosis concomitant with aberrant phospholipid remodeling in the hearts of lipodystrophic $B s c l 2^{-1-}$ mice.

Mechanisms underlying the drastic glycerolipid reduction in $B s c l 2^{-1-}$ hearts remain unknown. RNAseq identified no changes in the expression of genes involved in various aspects of cardiac fatty acid transport, glycerolipid, and glycerophospholipid metabolism (Supplemental Table 1), suggesting posttranscriptional mechanisms are involved. Interestingly, we found the expression of ATGL (gene name: Pnpla2), the rate-limiting cardiac TG hydrolase (11), was not altered at the mRNA level (Figure 2D), but elevated by approximately 2-fold at the protein level in hearts of 3-month-old $\mathrm{Bscl2^{-/ }}$ mice, whereas the expression of hormone-sensitive lipase (HSL, gene name Lipe) did not differ between 2 genotypes (Figure 2, E and F). 
A

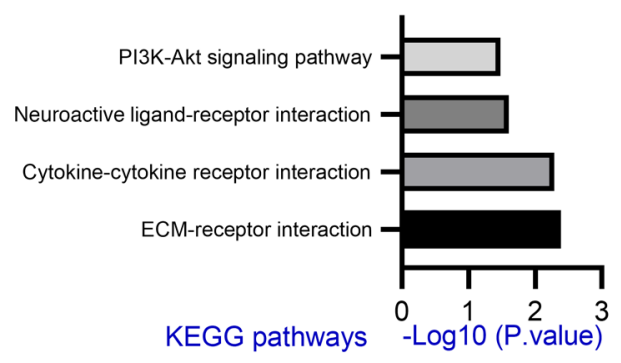

B

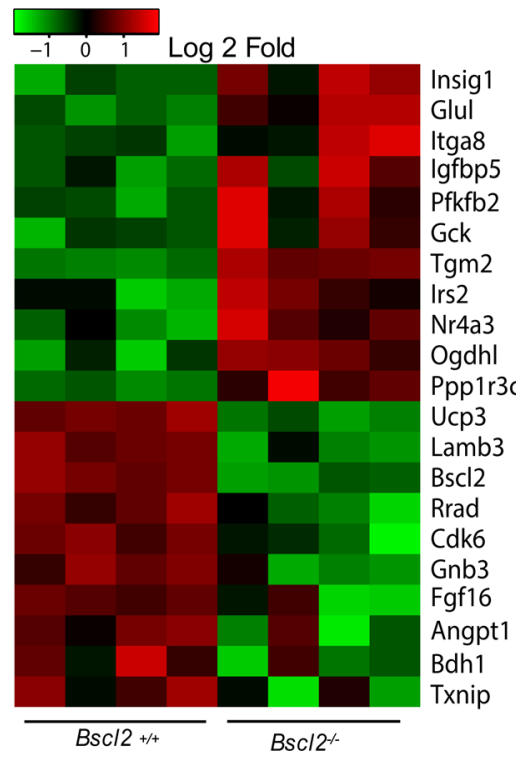

C
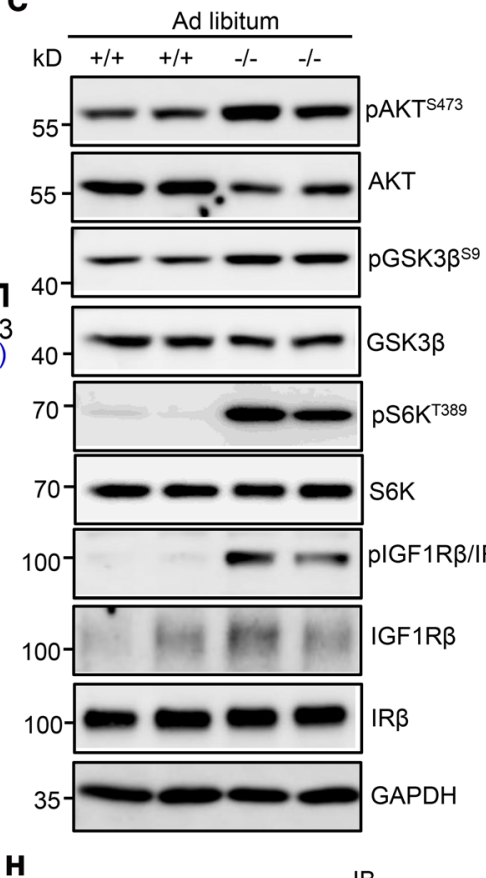

H

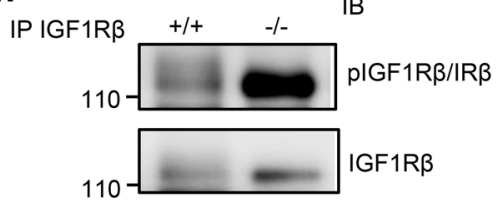

D
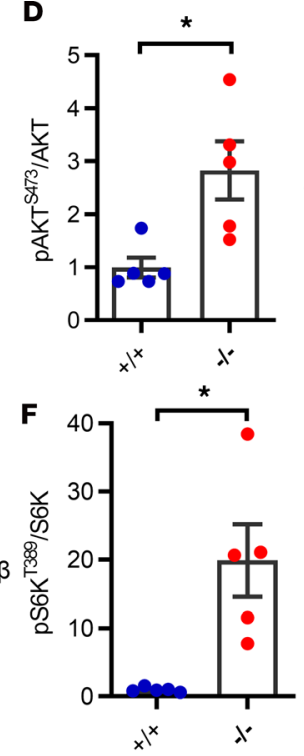

E
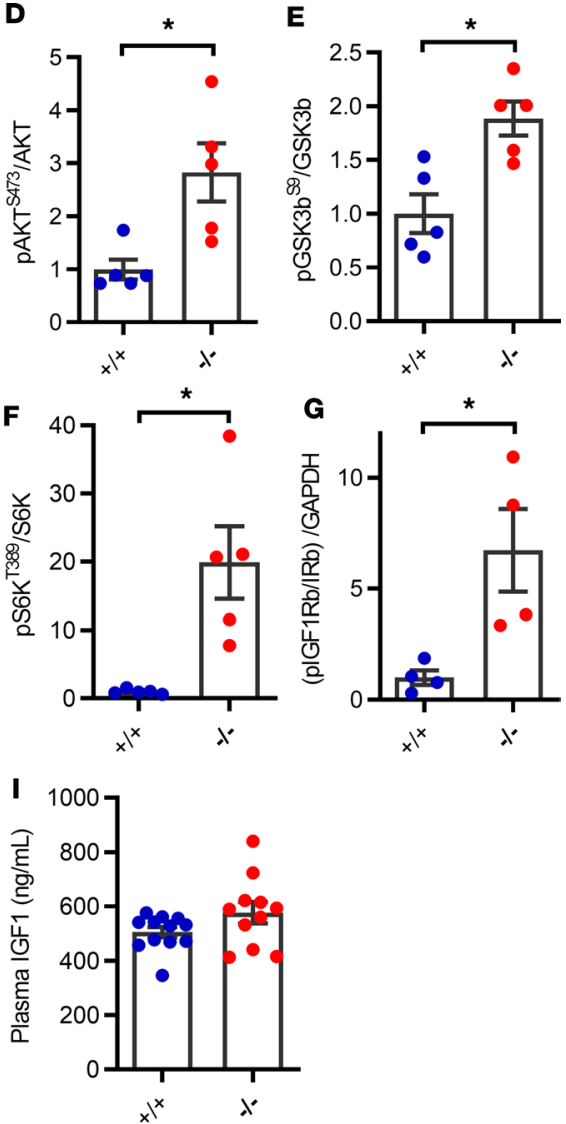

Figure 1. Elevated basal IGF1R-mediated PI3K/AKT signaling in hypertrophic Bsc/2-/- hearts. (A and B) Transcriptome and pathway analyses of differentially expressed (DE) genes and heatmap from DE genes related to PI3K/AKT signaling in left ventricles of 10-week-old female Bsc/2 ${ }^{-1-}$ mice after a 4-hour fast ( $n=4$ pooled from 3 animals each). (C-C) Western blot and quantification of pAKT at Ser473, pCSK3 $\beta$ at Ser9, pS6K at Thr389, and pIGF1R $\beta /$ IR $\beta$ at Tyr1158/Tyr1162/Tyr1163 in hearts ( $n=5$ /group). (H) Immunoprecipitation of cardiac IGF1R $\beta$ detects enhanced tyrosine phosphorylation of IGF1R $\beta$. Representative Western blot is shown. $n=5$ /group. (I) Plasma IGF1 levels ( $n=11 /$ group). For C-I, ad libitum-fed 3-month-old male $B s c 2^{+/+}$and $B s c / 2^{-/-}$mice were used. ${ }^{*} P<0.05$ by unpaired $t$ test.

Different from BSCL2-deleted adipose tissue (44), there were no major changes in cAMP/PKA-mediated phosphorylation in BSCL2-deleted hearts (Figure 2E). Consistent with the elevated ATGL protein expression, we identified an approximately 1.8-fold upregulation of total TG hydrolase activity in the hearts of $B s c l 2^{-1-}$ mice (Figure 2G). ATGL is a short-lived $\left(\mathrm{t}_{1 / 2} \sim 45\right.$ minutes) protein that is degraded through the ubiquitin proteasome pathway (45). Consistent with this, inhibition of proteasome function with bortezomib (BZM) led to significant accumulation of ATGL proteins in isolated adult cardiomyocytes (Figure 2H). Cycloheximide-based pulse-chase experiment revealed an elevated half-life of ATGL in BSCL2-deleted adult cardiomyocytes (Figure 2I). Similar findings were also observed in $B s c l 2^{-{ }^{-}}$mouse embryonic fibroblasts (MEFs) (Figure 2J). These data suggest that deletion of BSCL2 increases ATGL protein stability, contributing to enhanced intramyocellular TG turnover and thus myocardial glycerolipid remodeling in $\mathrm{Bscl2}^{-/-}$mice.

Elevated cardiac FAO and increased acetylation of mitochondrial $\beta$-oxidation enzymes in Bscl2 ${ }^{-1-}$ hearts. Expression of ATGL has been intimately linked to cardiac FAO and mitochondrial function (11). When analyzing mitochondrial $\beta$-oxidative capacity using crude heart homogenates, we identified the rates of complete oxidation of $\left[{ }^{14} \mathrm{C}\right]$ palmitate to $\mathrm{CO}_{2}$ in hearts of 3- and 6-month-old $\mathrm{Bscl2} 2^{-1-}$ mice were about $40 \%$ and $60 \%$ higher, respectively, compared with those of $\mathrm{Bscl}^{+/+}$mice (Figure $3 \mathrm{~A}$ ). The rates of radiolabel incorporation into acid-soluble metabolites (ASMs) in the hearts of 3- and 6-month-old $\mathrm{Bscl2}^{-/-}$mice were also elevated by about $25 \%$ and $50 \%$, respectively (Figure 3B). Seahorse electron flow assay detected either a tendency toward or higher oxygen consumption rates (OCRs) for mitochondrial electron transport chain 

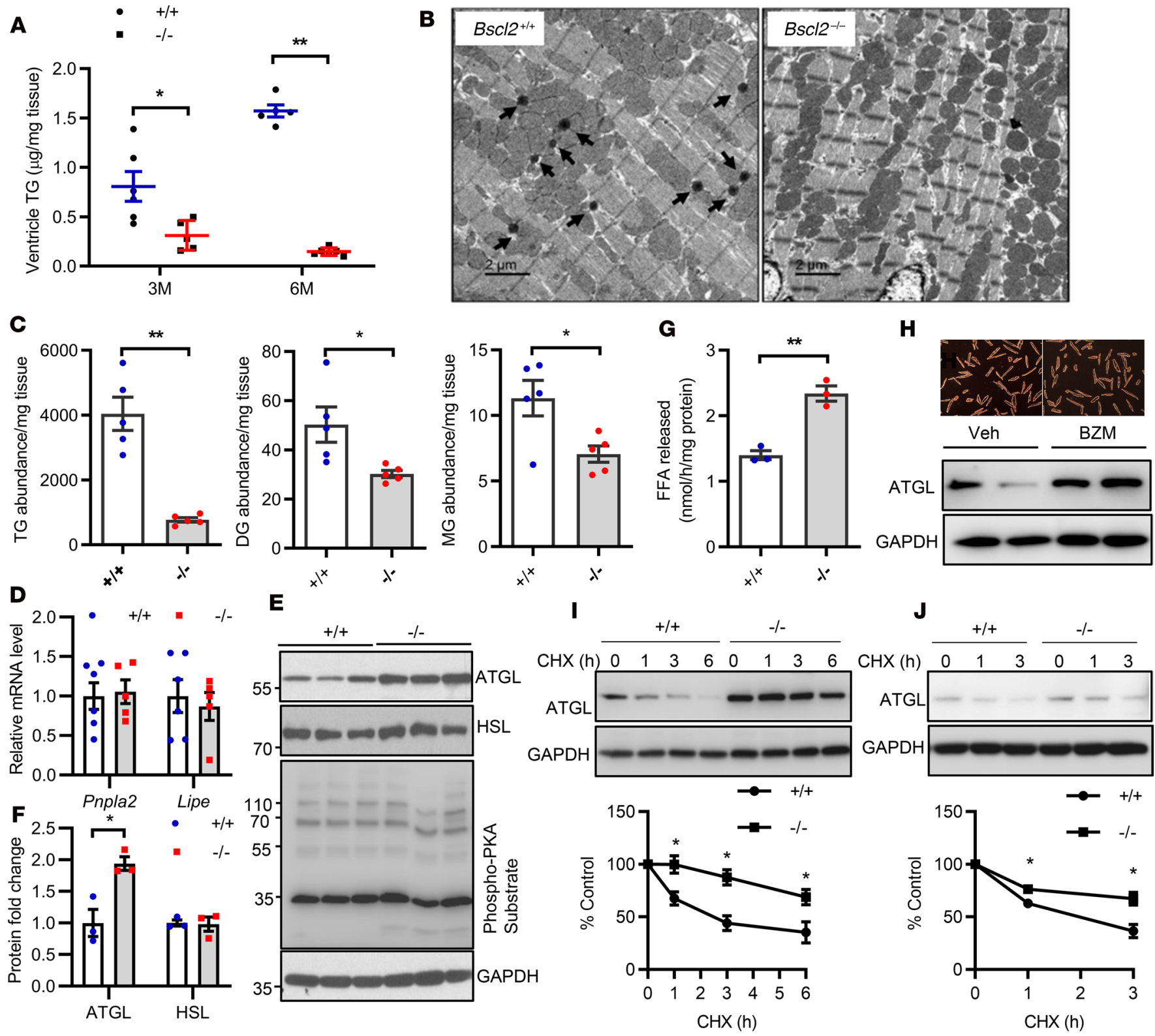

Figure 2. BSCL2 deficiency reduces intramyocellular glycerolipids and elevates ATGL stability and expression in the heart. (A) Quantitative enzymatic analyses of ventricle triglyceride (TG) in 3- and 6-month-old (3M and 6M) nonfasting Bscl2 ${ }^{+/+}(+/+)$and $B s c / 2^{-/-}(-/-)$mice (male, $\left.n=4-7 / g r o u p\right)$. (B) Representative transmission electron microscopy of 6-month-old nonfasting male Bscl2+/+ and $B s \mathrm{Cl} 2^{-/-}$hearts. Arrows indicate lipid droplets. Scale bars: $2 \mu \mathrm{m}$. (C) Comparison of the total normalized ion abundances for glycerolipids including TC, diacylglycerol (DG), and monoacylglycerol (MG) identified by lipidomics in hearts of 6-month-old male Bsc/2-/- mice fed ad libitum ( $n=5$ with each pooled from 3 animals). (D) RT-PCR analyses of Pnpla2 and Lipe gene expression in hearts of 3-month-old nonfasting mice ( $n=5-7 /$ group). (E and F) Representative Western blot and quantification of heart protein expression in 3-month-old male $B s c / 2^{+/+}$and $B s c / 2^{-/-}$mice fed ad libitum ( $n=3 /$ group). (C) TC hydrolase activity in 3-month-old heart homogenates incubated with radiolabeled ${ }^{3} \mathrm{H}$-triolein. Free fatty acid (FFA) release was measured and normalized to protein (male, $n=3$ in triplicate). (H) Viability and ATCL expression in primary adult mouse cardiomyocytes isolated from male [57BL/6) mice after incubation with vehicle (Veh) or 100 nM bortezomib (BZM) for 12 hours. (I and J) Cycloheximide (CHX) shutoff analysis of endogenous ATCL turnover in primary adult mouse cardiomyocytes isolated from 3-month-old male $B s c / 2^{+/+}$ and $\mathrm{Bscl} 2^{-/-}$mice and in $B s c / 2^{+/+}$and $B s c / 2^{-/-}$MEFs. Densitometry from Western blots was standardized to ATCL expression at 0 hours. ${ }^{*} P<0.05$; ${ }^{*} P<$ 0.005 by unpaired $t$ test (C and $\mathbf{G}$ ) or multiple $t$ tests after correction using the Holm-Sidak method (A, D, F, I, and J).

(ETC) complexes I-IV in isolated mitochondria from the hearts of 3-month-old $\mathrm{Bscl2} \mathrm{2}^{-1}$ mice compared with $\mathrm{Bscl2}^{+/+}$mice (Figure 3C). Elevated FAO rates and mitochondrial respiration were not associated with differences in mitochondrial biogenesis based on similar mtDNA copies/nucleus (Supplemental Figure $3 \mathrm{~A}$ ), the transcriptional expression of genes involving FAO and ETC components (Supplemental Table 1), and the expression of mitochondrial complex proteins between 2 genotypes (Supplemental Figure 3B). 
Cardiac protein acetylation has been shown to control activities of enzymes involved in mitochondrial FAO and respiration in hearts from high-fat diet-induced obese mice (14). Interestingly, we found a significant increase in overall lysine acetylation of cardiac proteins without downregulation of SIRT3, the main mitochondrial deacetylase (46) in both whole-heart homogenates (Figure 3, D and E) and isolated cardiac mitochondria (Figure 3F) in 3- and 6-month-old Bscl2 ${ }^{-1-}$ mice. More specifically, despite no changes in its protein expression, lysine acetylation of long-chain acyl-CoA dehydrogenase (LCAD), one of the key FAO enzymes, was significantly increased (Figure 3G) and positively correlated to an elevated LCAD activity (Figure $3 \mathrm{H}$ ) in 6-month-old $\mathrm{Bscl}^{-/-}$hearts. Thus, ATGL upregulation and hyperacetylation of FAO enzymes may contribute to the excessive FAO in hearts of BSCL2 lipodystrophy.

Despite excessive FAO, we did not identify increased oxidative stress by $2^{\prime}, 7^{\prime}$-dichlorofluorescein diacetate (DCFDA) staining and direct measurement of the levels of peroxidative product malondialdehyde (MDA) in 6-month-old Bscl2 ${ }^{-1-}$ hearts (Supplemental Figure 3, C and D), consistent with previous findings (4). Notably, the protein expression of catalase (CATA) but not SOD2 was consistently higher in 3- and 6-month-old $\mathrm{Bscl2}^{-/-}$hearts (Supplemental Figure 3E), which may represent an important adaptive antioxidant response to prevent oxidative damage. Moreover, accumulation of toxic lipid intermediates, such as sphingomyelins and ceramides in $B s c l 2^{-1-}$ hearts (Supplemental Figure 3F), was not detected in association with increased translocation of $3 \mathrm{PKC}$ isoforms (PKC $\varepsilon,-\delta$, and $-\zeta)$ to the membrane in $\mathrm{Bsl2}^{-/-}$ventricles compared with those of $\mathrm{Bscl}^{+/+}$fractions (Supplemental Figure 3G). However, measurement of glucose oxidation capacity in crude heart homogenates did not identify a significant difference between 2 genotypes in 3-month-old hearts, but there was a tendency toward a lower glucose oxidation rate in 6-month-old $\mathrm{Bscl2^{-/ }}$ hearts, in line with a gradual decreased cardiac function (Supplemental Figure $3 \mathrm{H}$ ). These data suggest that $B s c l 2^{-/}$hearts exhibit excessive $\mathrm{FAO}$ and mitochondrial respiration, leading to cardiac substrate shift and dysfunction in $\mathrm{Bscl}^{-/-}$mice.

Loss of ATGL dose-dependently reverts BSCL2 lipodystrophy and its associated metabolic disturbances. The lack of functional adipose tissue has been known to cause severe metabolic diseases, such as insulin resistance, hepatomegaly, and diabetes mellitus in BSCL. Previously, we have demonstrated uncontrolled cAMP/ PKA-mediated lipolysis as the culprit for the aborted adipocyte differentiation and ultimately lipodystrophy in $B s c l 2^{-1-}$ mice (23). Activation of $\mathrm{CAMP} / \mathrm{PKA}$ signaling recruits more ATGL to LDs to stimulate lipolysis in differentiating BSCL2-deleted adipocytes (23). Upregulation of ATGL at the protein level was also observed in $\mathrm{Bscl2}^{---}$hearts, in association with reduced intramyocellular TG storage (Figure 2). These data suggest that BSCL2 deficiency acts through differential pathways to upregulate ATGL-mediated TG hydrolysis in adipose tissue and the heart. On the basis of these findings, we next tested whether targeting ATGL could rescue lipodystrophy and its associated cardiac dysfunction. We generated $B s c l 2^{-1-}$ mice with partial and complete deletion of ATGL. Interestingly, partial genetic inactivation of ATGL by deletion of 1 allele in $B s c l 2^{--}$mice (denoted as $A^{h} B^{k}$ mice) could restore approximately $30 \%$ of fat mass compared with global lipodystrophic $B s c l 2^{--}\left(A^{w} B^{k}\right)$ mice, whereas complete ablation of ATGL in $B s c l 2^{-1-}$ mice $\left(A^{k} B^{k}\right)$ fully rescued lipodystrophy, with percentage fat mass even greater than that in wild-type $\left(A^{w} B^{w}\right)$ mice, albeit lower than that in ATGL-single-knockout $\left(A^{k} B^{w}\right)$ mice (Figure 4A). No differences in body weights were observed across the 5 groups (Supplemental Figure 4A). Organ weights of gonadal white adipose tissue (gWAT) (Figure 4B) were restored by 4 -fold and 17-fold in $A^{h} B^{k}$ and $A^{k} B^{k}$ mice, respectively, compared with lipodystrophic $A^{w} B^{k}$ mice, with similar changes in subcutaneous WAT (sWAT) masses (Figure 4C). Morphological analyses of gWAT and sWAT further supported the findings, with $A^{h} B^{k}$ and $A^{k} B^{k}$ mice demonstrating allele-dependent increases of adipose tissue volume in comparison with $A^{w} B^{k}$ mice (Figure 4D). $\mathrm{H} \& \mathrm{E}$ staining of gWAT and sWAT further demonstrated expansions of adipocyte size and lipid accumulation in $A^{h} B^{k}$ mice, whereas the LD sizes and adipocyte distribution in $A^{k} B^{k}$ mice phenocopied those in $A^{w} B^{w}$ and $A^{k} B^{w}$ mice (Figure 4E). As expected, ATGL expression was partially reduced in gWAT of $A^{h} B^{k}$ mice and completely absent in gWAT of both $A^{k} B^{k}$ and $A^{k} B^{w}$ mice. The largely reduced expression of ATGL in the residual gWAT of $A^{w} B^{k}$ mice could be secondary to lipodystrophy. The expression of adipocyte marker protein PLIN1 did not vary among each genotype. However, the expression of PLIN2, an LD protein consistently upregulated in BSCL2-deleted WAT (23), maintained its upregulation in $A^{w} B^{k}$ and $A^{h} B^{k}$ mice, but was greatly reduced in gWAT of $A^{k} B^{k}$ mice to a level comparable to that in $A^{w} B^{w}$ mice (Figure $4 \mathrm{~F}$ ). These data highlight that ATGL is downstream of BSCL2 that governs the differentiation of $B s c l 2^{-1-}$ adipocytes in vivo.

To our surprise, heterozygous deletion of ATGL almost fully reversed hyperinsulinemia (Figure $4 \mathrm{G}$ ) and partially reduced hepatomegaly compared with $A^{w} B^{k}$ mice (Supplemental Figure 4B). 

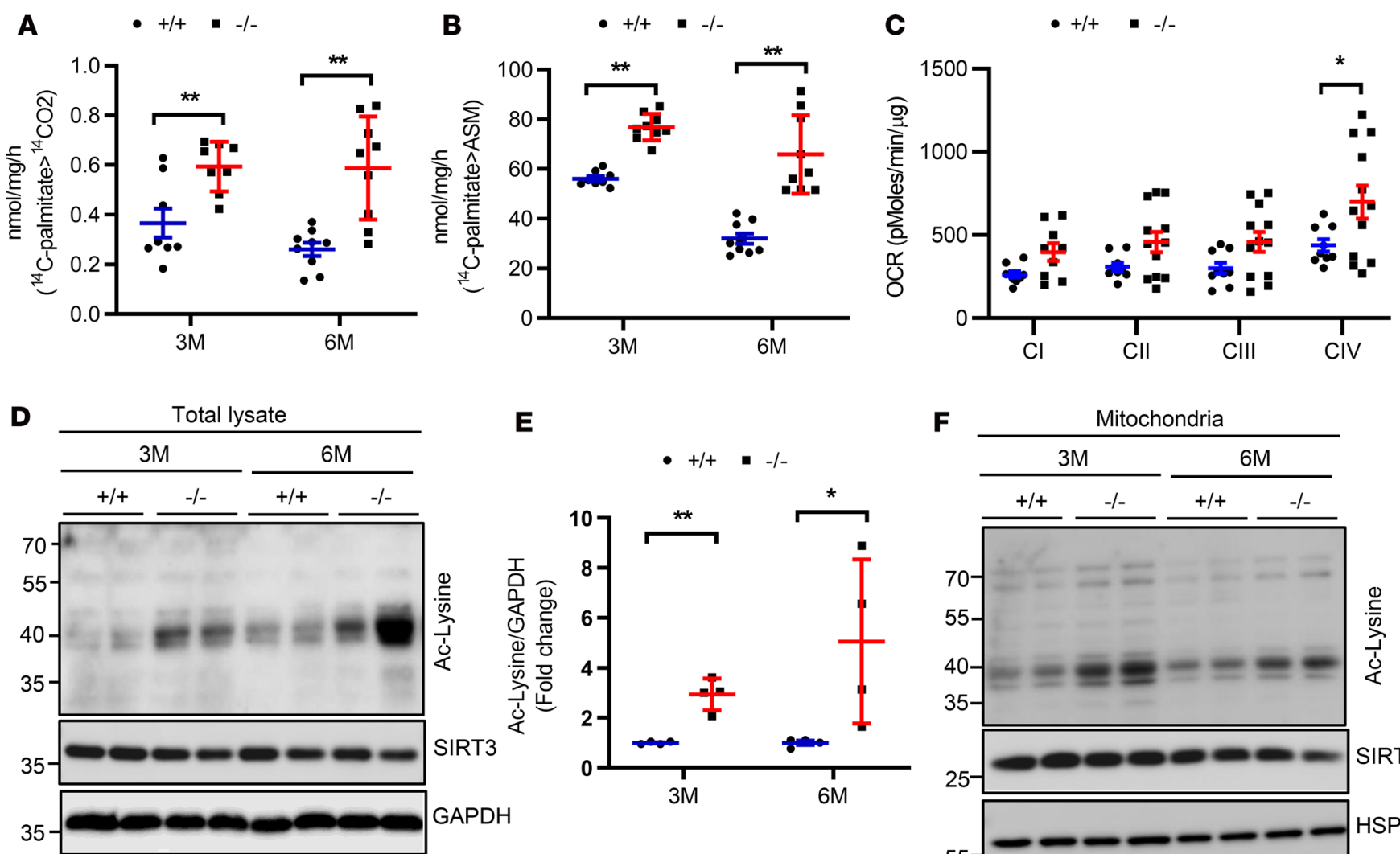

E
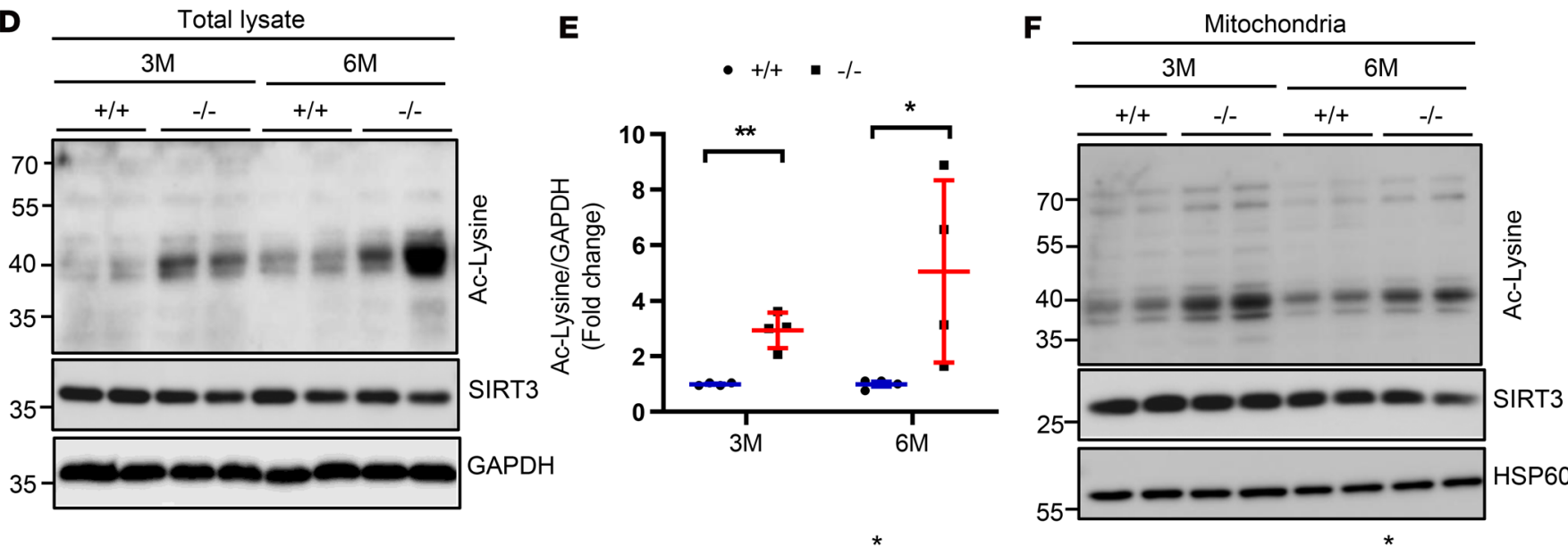

G
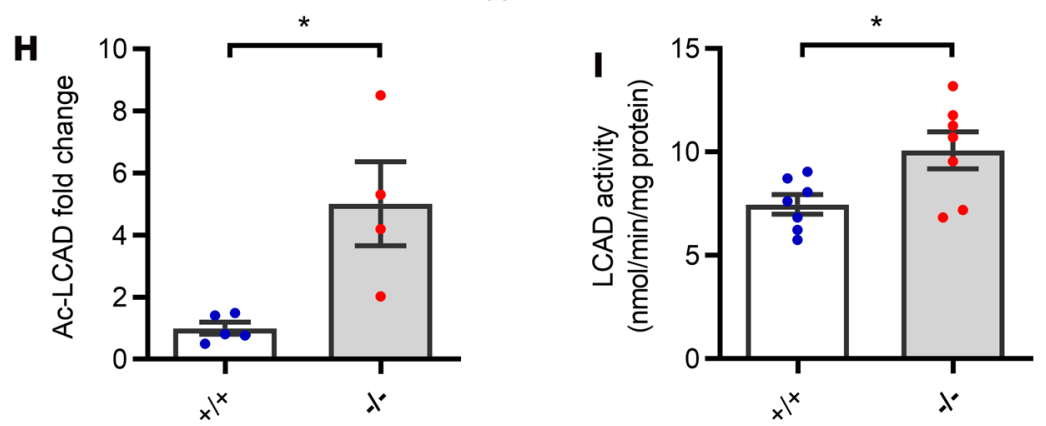

Figure 3. Bscl2-/- mice display excessive cardiac fatty acid oxidation and hyperacetylation. (A and B) $\mathrm{CO}_{2}$ and acid soluble metabolite (ASM) production after incubating heart crude mitochondrial fraction with ${ }^{14} \mathrm{C}$-palmitate (male, $n=6 /$ group in triplicate). (C) Measurements of oxygen consumption rates (OCRs) to assess respiratory chain activities of mitochondrial complex I to IV (CI to CIV) by electron flow assays. Mitochondria were isolated from 3-monthold (3M) male Bscl2 $2^{++}(+/+)$and $B s c / 2^{-/-}(-/-)$mice. $n=4 /$ group in triplicate. (D and E) Representative Western blot and quantification of total protein acetylation in hearts from 3-and 6-month-old male mice ( $n=4$ /group). (F) Representative Western blot of protein acetylation in isolated mitochondria from hearts of 3- and 6-month-old male mice ( $n=4$ /group). ( $\mathbf{G}$ and $\mathbf{H}$ ) Level of acyl-CoA dehydrogenase long chain (LCAD) acetylation. Total lysates from hearts of 6-month-old male $B s c / 2^{+/+}$and $B s c / 2^{-1-}$ mice were immunoprecipitated with anti-acetylated lysine (anti-Ac-Lysine) antibody and immunoblotted with antibody specific for LCAD ( $n=4-5 /$ group). (I) LCAD activity in hearts of 6-month-old male $B s c / 2^{+/+}$and $B s c / 2^{-/-}$mice $\left(n=7 /\right.$ group). ${ }^{*} P<0.05$; ${ }^{* *} P<$ 0.005 by multiple $t$ tests after correction using the Holm-Sidak method ( $\mathbf{A}-\mathbf{C}$ and $\mathbf{E}$ ) or unpaired $t$ test (H and $\mathbf{I})$.

Although 10-week-old $A^{w} B^{k}$ mice did not exhibit overt hyperglycemia after a 4-hour fast, the fasting plasma glucose level was lower in $A^{k} B^{k}$ mice than $A^{w} B^{w}$ mice (Supplemental Figure 4C). As expected, the extremely lower leptin level in lipodystrophic $A^{w} B^{k}$ mice was moderately upregulated to a similar extent by both heterozygous and homozygous deletion of ATGL (Supplemental Figure 4D). Most importantly, loss of ATGL in Bscl2 ${ }^{--}$mice improved whole-body insulin sensitivity, with the insulin sensitivity of $A^{k} B^{k}$ mice completely restored to that of $A^{w} B^{w}$ mice (Figure $4, \mathrm{H}$ and I). These data highlight a striking rescue of lipodystrophy and its associated insulin resistance and hepatomegaly even with heterozygous ATGL deletion in BSCL2 lipodystrophy.

Pharmacological and genetic inhibition of ATGL partially rescues adipocyte differentiation of Bscl2-- cells in vitro. To further determine whether ATGL deletion-mediated restoration of adipose mass in $\mathrm{Bscl2}^{-1}$ 
A

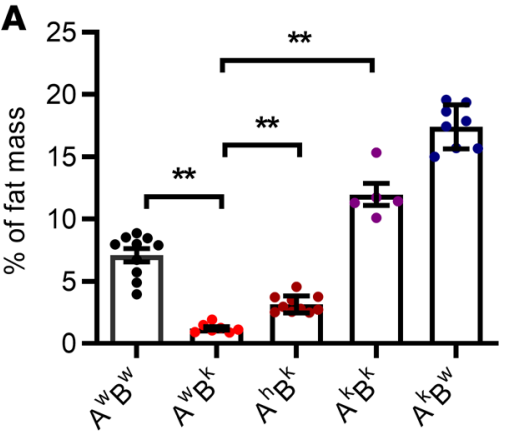

D $\quad A^{w} B^{w} \quad A^{w} B^{k} \quad A^{h} B^{k}$
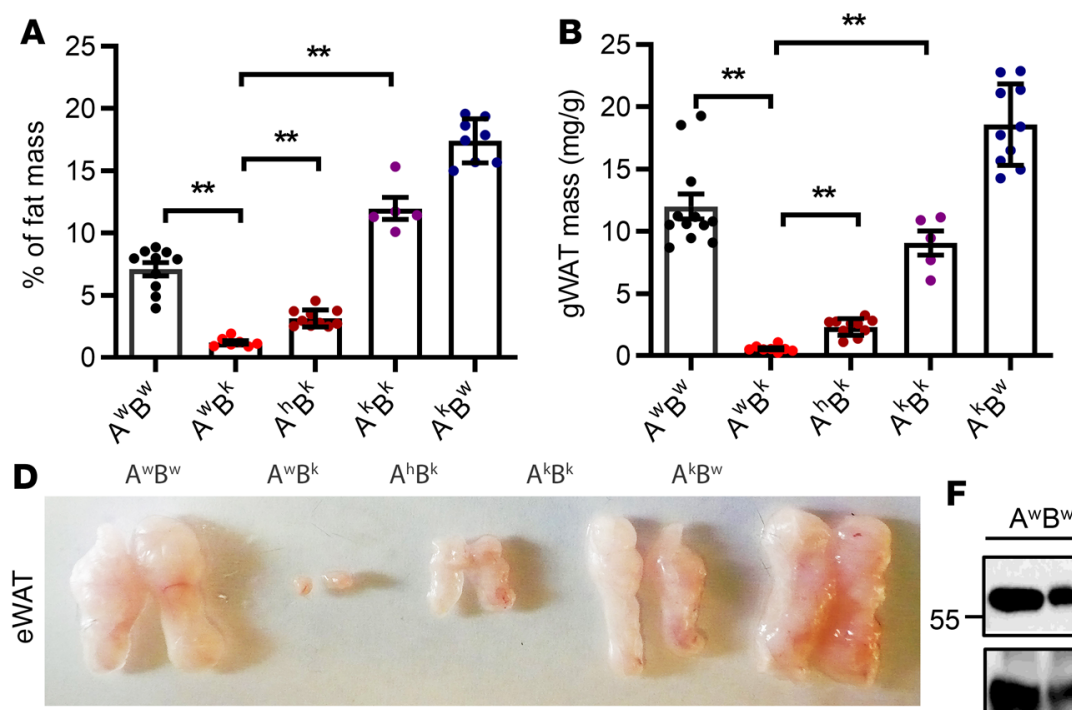

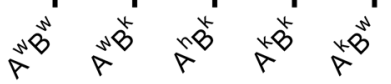

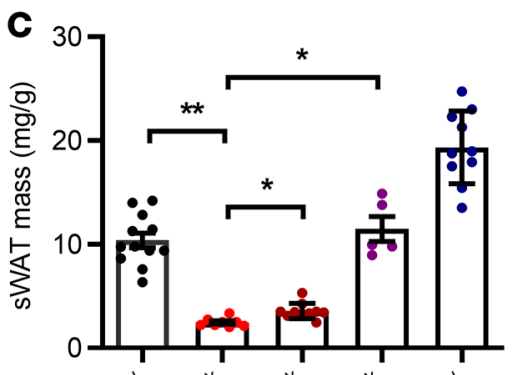

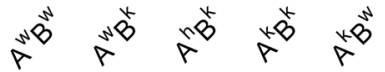
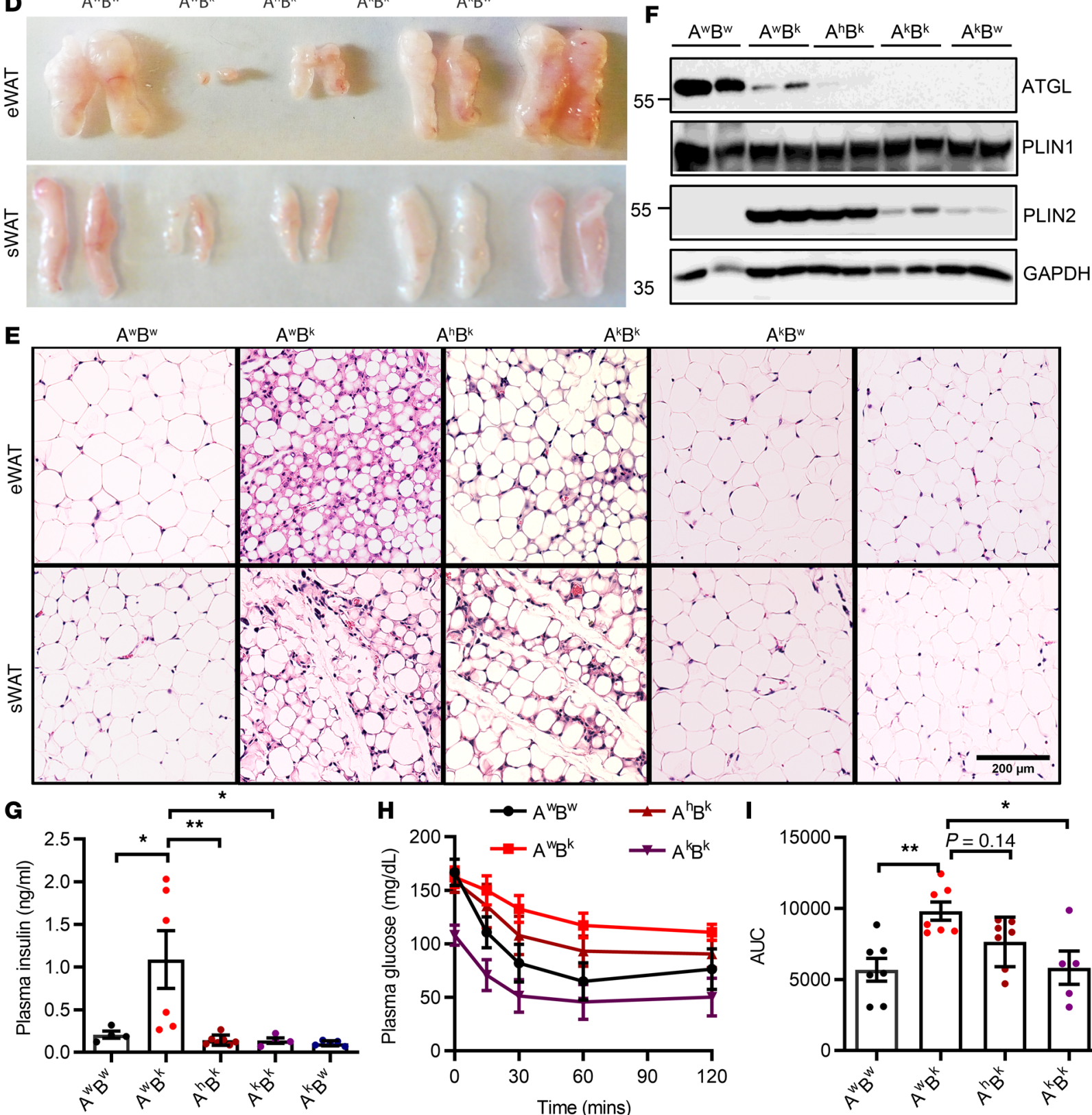

$A^{k} B^{w}$

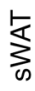

$A^{h} B^{k} \quad A^{k} B^{k}$
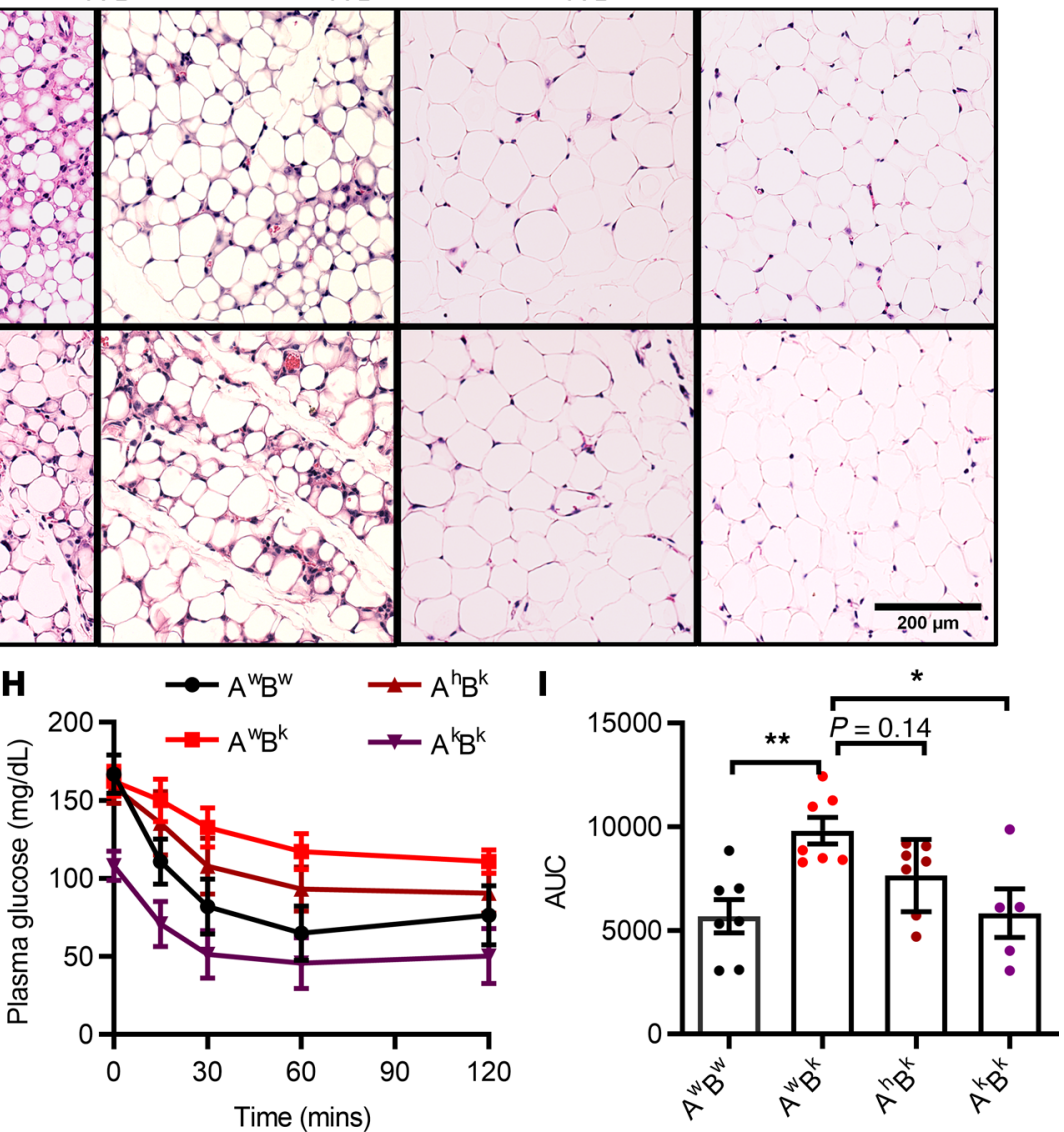

Figure 4. ATGL ablation rescues lipodystrophy and its associated insulin resistance in Bscl2-/- mice. (A-C) Percentage of fat mass assessed by NMR, and masses of gonadal white adipose tissue (gWAT) and subcutaneous WAT (sWAT) as normalized to body weight. (D and E) Representative images and H\&E staining of gWAT and sWAT. Scale bar: $200 \mu \mathrm{m}$. (F) Representative Western blotting of mature adipose marker and lipid droplet protein (LDP). (G)

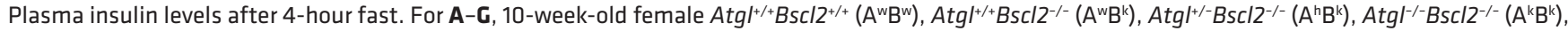
and $A t g 1^{-1-} B s c / 2^{+/+}\left(A^{k} B^{w}\right)$ littermates were used for all experiments ( $n=5-10 /$ group). Limited numbers of male $A^{k} B^{k}$ mice were obtained. However, a similar extent of rescue of lipodystrophy was observed in male $A^{\mathrm{h}} \mathrm{B}^{\mathrm{k}}$ and $A^{\mathrm{k}} \mathrm{B}^{\mathrm{k}}$ mice. ( $\mathbf{H}$ and $\left.\mathbf{I}\right)$ Insulin tolerance test and area under the curve (AUC) in mice other than $\mathrm{A}^{\mathrm{k}} \mathrm{B}^{\mathrm{w}}$ (male and female, $n=5-7 /$ group). ${ }^{*} P<0.05 ;{ }^{* *} P<0.005$ by 1 -way ANOVA with Dunnett's multiple-comparisons correction. 
mice occurs autonomously, we first treated differentiating $\mathrm{Bscl2}^{+/+}$and $\mathrm{Bscl}^{-{ }^{--}}$MEFs with Atglistatin (a specific ATGL inhibitor) (47) from 4 to 10 days (D4-D10) after induction of adipogenic differentiation as previously described (23). As expected, Atglistatin treatment prevented $\mathrm{Bscl}^{-{ }^{--}}$cells from abortion of adipocyte differentiation as indicated by an increased number of cells with Oil Red $\mathrm{O}$ (ORO) and LipidTOX staining (Figure 5A), elevated intracellular TG content (Figure 5B), as well as enhanced protein expression of adipocyte markers such as PPAR $\gamma$ and PLIN1 at D10 (Figure 5C). When isolating stromal vascular cells (SVCs) from sWAT of $A^{w} B^{w}, A^{w} B^{k}$, and $A^{k} B^{k}$ mice for adipocyte differentiation, we observed almost intact upregulation of Ppary and Plin1 mRNAs in $A^{w} B^{k}$ and $A^{k} B^{k}$ cells at D4 after adipocyte differentiation compared with $A^{w} B^{w}$ cells. However, by D10, expression of those marker proteins was drastically lower in $A^{w} B^{k}$ cells than in wild-type SVCs, again suggesting abortion of the adipocyte differentiation program in the absence of BSCL2. Interestingly, the expression of Ppary and Plin1 in $A^{k} B^{k}$ cells at D10 was about 20-fold higher than $A^{w} B^{k}$ cells, albeit much lower than $A^{w} B^{w}$ cells (Figure 5D). Western blot also revealed a modest upregulation of PLIN1 in D10 $A^{k} B^{k}$ adipocytes compared with $A^{w} B^{k}$ cells (Figure 5E). Partial rescue of $B s c l 2^{-1-}$ adipocyte differentiation by ATGL deletion was also confirmed by ORO staining and intracellular TG content (Figure 5, F and G, respectively). These data highlight that pharmacological inhibition or genetic inactivation of ATGL rescues the defects in adipocyte differentiation caused by loss of BSCL2.

Partial inactivation of ATGL ameliorates cardiac hypertrophy, metabolic inflexibility, and cardiac dysfunction in $\mathrm{Bscl}^{-1-}$ mice. Complete ATGL ablation in mice causes severe cardiac steatosis and lethal cardiomyopathy by 12 weeks of age (48). Because generating a small mass of functional adipose tissue in $A^{h} B^{k}$ mice was sufficient to reverse the whole-body insulin resistance of $\mathrm{Bscl2}^{-/-}$mice, we next focused on whether partial inactivation of ATGL could ultimately improve cardiac dysfunction manifested in 6-month-old $B s c l 2^{-1-}$ mice. Indeed, 6-month-old $A^{h} B^{k}$ mice developed hyperinsulinemia (Figure 6A) and hyperglycemia (Supplemental Table 2), but the levels were significantly attenuated compared with $A^{w} B^{k}$ mice. Cardiac hypertrophy in $A^{h} B^{k}$ mice was almost completely reversed (Figure 6B), which correlated well with attenuated activation of prohypertrophic PI3K/AKT and its downstream signaling (Supplemental Figure 5A). Not surprisingly, echocardiographic assessment of LV function revealed augmented fractional shortening (Figure 6C) and ejection fraction (Supplemental Table 2), as well as a small but not statistically significant reduction in left ventricle internal diameter at systole (Figure 6D) in $A^{h} B^{k}$ mice compared with $A^{w} B^{k}$ mice, suggesting rescue of cardiac function.

As expected, partial ATGL deletion in $B s c l 2^{-1-}$ mice abolished cardiac ATGL upregulation (Figure $6 \mathrm{E}$ ), which contributed to a minimal upregulation of cardiac TG content compared with $A^{w} B^{k}$ hearts (Supplemental Table 2). Interestingly, the level of cardiac protein acetylation was approximately $50 \%$ lower in $A^{h} B^{k}$ hearts than $A^{w} B^{k}$ hearts (Figure 6, E and F), as was the mitochondrial protein acetylation (Supplemental Figure 5B). Accordingly, elevated LCAD activity in $A^{w} B^{k}$ hearts was significantly mitigated in $A^{h} B^{k}$ hearts to a similar level as observed in $A^{w} B^{w}$ hearts (Figure 6G). Homogenates of $A^{h} B^{k}$ mouse hearts exhibited a tendency toward lower FAO rates than those of $A^{w} B^{k}$ mice, as indicated by ASM production from ${ }^{14} \mathrm{C}$-palmitate (Supplemental Figure $5 \mathrm{C}$ ). Likewise, mitochondrial activity in $A^{h} B^{k}$ hearts was also ameliorated to a comparable level as seen in $A^{w} B^{w}$ hearts (Figure 6H). Most importantly, alleviation of excessive FAO was accompanied with an improved glucose oxidation in $A^{h} B^{k}$ hearts (Figure 6I), suggesting a refined substrate flexibility. These changes were also correlated to an improved cardiac insulin sensitivity in response to insulin in hearts of $A^{h} B^{k}$ mice compared with $A^{w} B^{k}$ mice (Supplemental Figure 5, D and E). Together, these data highlight that whole-body partial ATGL deletion improves cardiac substrate flexibility and restores cardiac function in lipodystrophic $\mathrm{Bscl}^{-/-}$mice.

\section{Discussion}

Our study provided a number of insights into the pathophysiology of BSCL2 lipodystrophy and its associated cardiac dysfunction. We first demonstrated a critical role for IGF1R-mediated PI3K/AKT signaling in promoting cardiac hypertrophy in BSCL2 lipodystrophy. Secondly, we identified a pattern of cardiac lipid remodeling in a nonobese insulin-resistant animal model with reduced cardiac steatosis associated with ATGL overexpression. Thirdly, BSCL2 lipodystrophy induces cardiac mitochondrial protein hyperacetylation, excessive FAO, mitochondrial hyperactivation, and ultimately cardiac dysfunction. Most importantly, we found ATGL haploinsufficiency could reverse BSCL2 lipodystrophy and its associated insulin resistance and ultimately normalize cardiac derangements. 

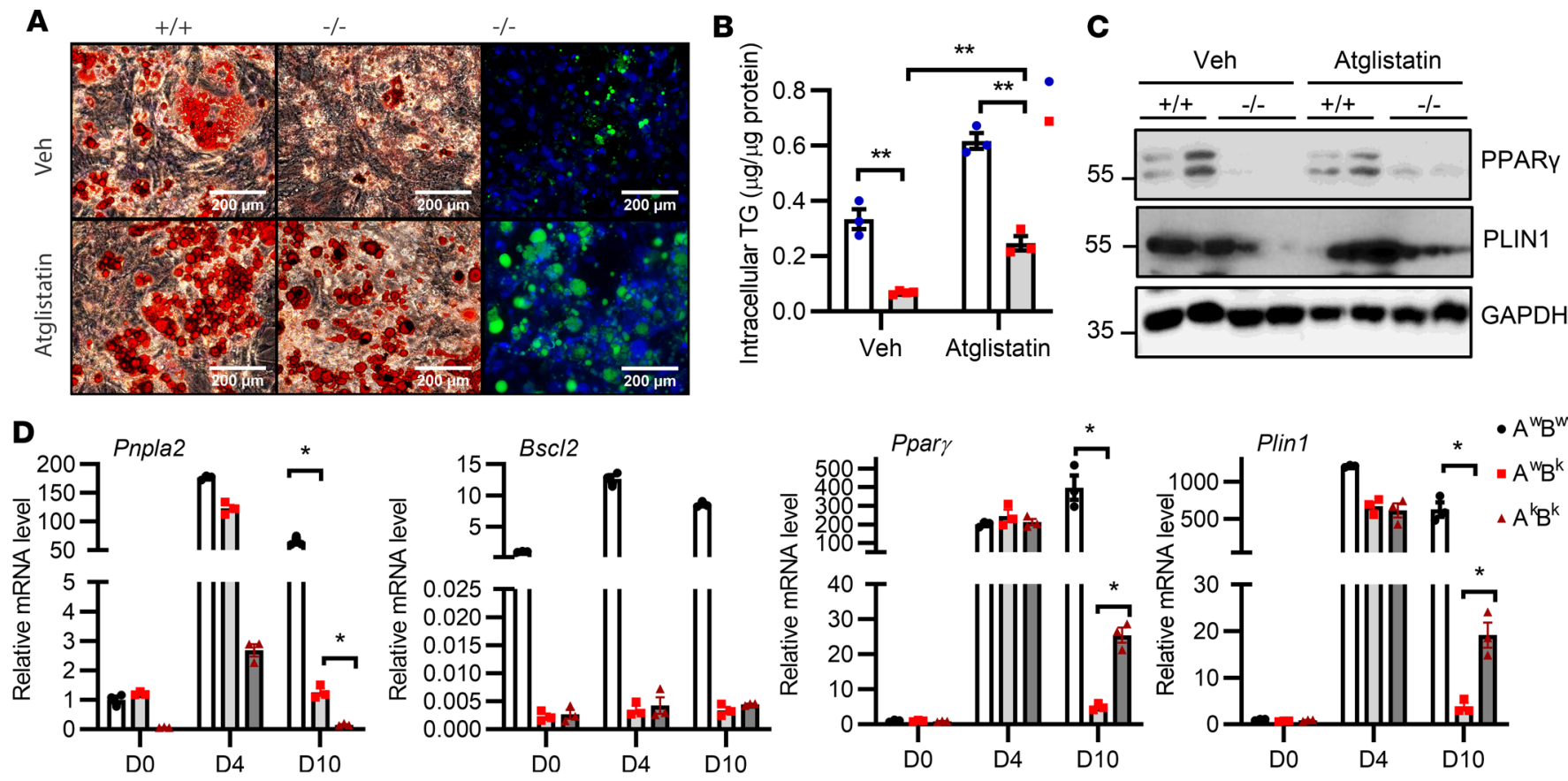

$\mathbf{E}$

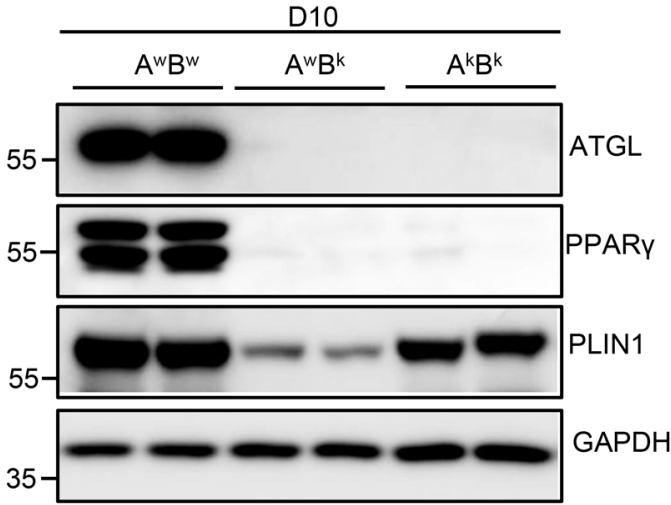

$\mathbf{F}$

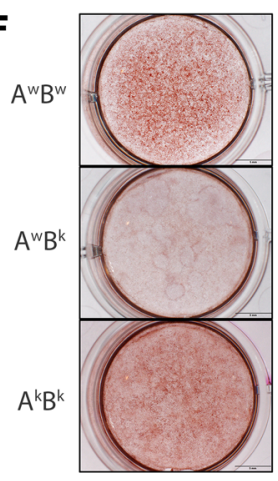

G

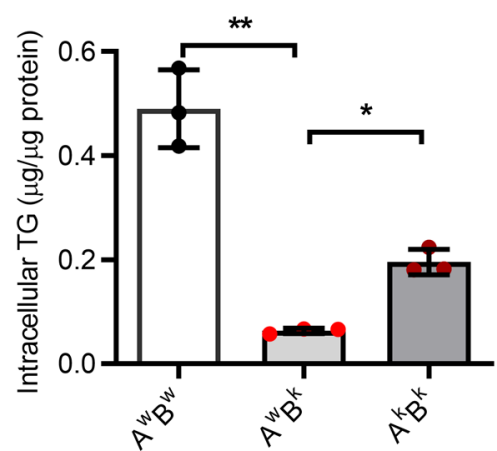

Figure 5. ATGL inhibition partially rescues adipocyte differentiation of $\mathbf{B s c / 2} \mathbf{2}^{-/-}$cells. $B s c / 2^{+/+}$and $B s c / 2^{-/-}$MEFs were subjected to a standard hormone cocktail DMI (dexamethasone, IBMX, and insulin) to induce adipocyte differentiation. Four days (D4) after differentiation, cells were treated with vehicle (Veh) or $10 \mu \mathrm{M}$ Atglistatin and kept until D10. (A) Oil Red O and HCS LipidTOX Green neutral lipid staining. Scale bars: $200 \mu \mathrm{m}$. (B) Intracellular triglyceride (TC) content (2-way ANOVA with post hoc Tukey's test) and (C) representative Western blot of PPAR $\gamma$ and PLIN1 at D10 after DMI induction. (D-C) Stromal vascular cells isolated from $A t g I^{+++} B s c / 2^{+/+}\left(A^{w} B^{w}\right), A t g I^{++} B s c / 2^{-/-}\left(A^{w} B^{k}\right)$, and $A t g 1^{-/-} B s c / 2^{-/-}\left(A^{k} B^{k}\right)$ mice were subjected to DMI-induced adipocyte differentiation. (D) mRNA expression of Pnpla2, Bscl2, Ppary, and Plin1 was measured at D0, D4, and D10 after DMI induction (2-way ANOVA with Dunnett's multiple-comparisons post-hoc correction). (E) Representative protein expression, (F) Oil Red 0 staining, and (G) intracellular TC content at D10 after adipocyte induction (1-way ANOVA with Dunnett's correction for multiple comparisons). ${ }^{*} P<0.05 ;{ }^{* *} P<0.005$.

Adipocyte dysfunction is at the origin of the metabolic complications associated with lipodystrophy and adipose tissue transplantation has been shown to strongly improve the metabolic phenotype of lipodystrophic mice (49). Our study identified a means to restore functioning adipose tissue and treat metabolic diseases in BSCL2 lipodystrophy. Genetic evidence derived from our in vivo systems rigorously established ATGL as a physiologically relevant, bona fide downstream target of BSCL2 (Figure 4). To our knowledge, this is the first evidence at the organismal level that BSCL2 controls adipogenesis at least in part by regulating ATGL-mediated lipolysis in vivo. Notably, no direct interaction of BSCL2 with ATGL has been identified. Loss of ATGL alone has no impact on adipocyte differentiation (48). However, the fact that pharmacological inhibition or genetic inactivation of ATGL could partially rescue the defects in adipocyte differentiation caused by loss of BSCL2 supports our original finding (23) and emphasizes the notion that BSCL2 deletion causes rampant cAMP/PKA/ATGL-mediated lipolysis to abort adipocyte differentiation. These data not only provide insights into BSCL2 function but also the pathway controlling adipocyte differentiation. Overall, our findings 

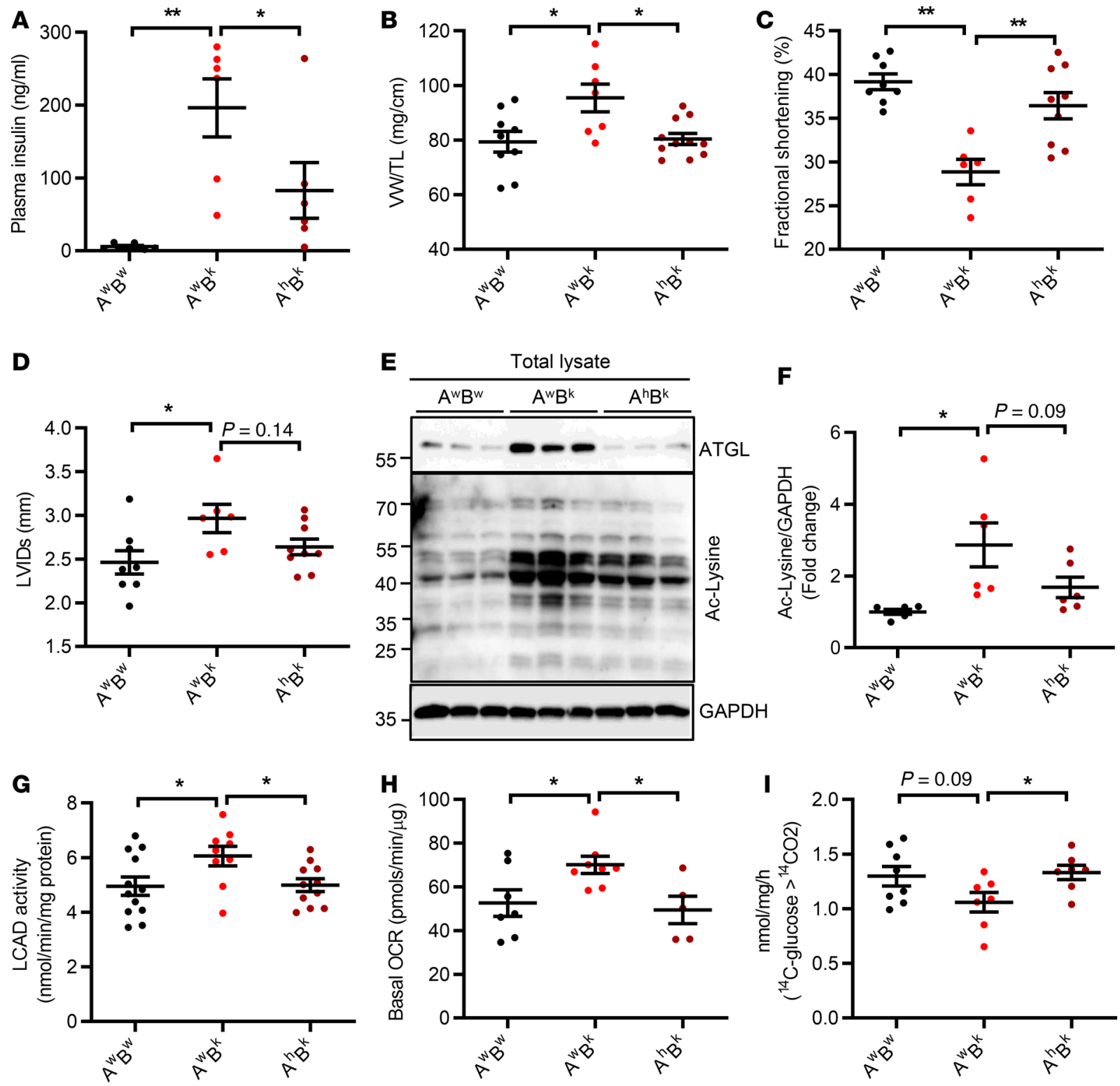

Figure 6. Partial ATGL inactivation rescues hypertrophy and cardiomyopathy in Bsc/2 ${ }^{-/-}$mice. (A) Nonfasting plasma insulin levels, (B) ventricle weight (VW) normalized to tibia length (TL), and (C and D) fractional shortening (\%) and left ventricle internal diameter at systole (LVIDs, mm). (E and F) Representative Western blotting (E) and fold changes of acetylated lysine (Ac-Lysine) as normalized to GAPDH (F) in whole heart. (C) Cardiac LCAD activity, (H) basal oxygen consumption rate (OCR) in isolated cardiac mitochondria, (I) $\mathrm{CO}_{2}$ production after incubating heart crude mitochondrial fraction with

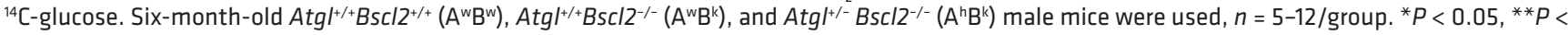
0.005 by one-way ANOVA with Dunnett's multiple-comparisons correction.

furthered our understanding of the pathophysiology of BSCL2 lipodystrophy, which could potentially provide the first strategy to address the lack of functional adipose tissue in BSCL2 lipodystrophy.

Lipodystrophy is widely associated with organomegaly, although to our knowledge the underlying mechanisms have never been interrogated in an animal model of lipodystrophy. Using global RNA profiling and biochemical analyses, we demonstrate that activation of prohypertrophic IGF1R-mediated PI3K/AKT signaling contributes to cardiac hypertrophy in $\mathrm{Bscl}^{-/-}$mice. The fact that higher IGF1R/PI3K/AKT signaling occurred in the absence of elevated circulating IGF1 level provides the first evidence to our knowledge that lipodystrophyinduced hyperinsulinemia may enable insulin to spill over to activate the IGF1R, thus stimulating organ growth. In support of this notion, reduction of hyperinsulinemia in $A^{h} B^{k}$ mice was associated with reduced prohypertrophic PI3K/AKT signaling and cardiac hypertrophy (Figure 6, A and B, and Supplemental Figure 5A). Thus, our data provide an important link between hyperinsulinemia and organomegaly in lipodystrophic $\mathrm{Bscl2}^{-1-}$ mice. 
Conflicting clinical reports found either lack of fatty infiltration $(18,50)$ or elevated TG content $(51)$ in the hypertrophied hearts of BSCL patients. Ventricles of $\mathrm{Bscl2}^{-1-}$ mice were previously identified to contain comparable TG and reduced DG contents (4). In contrast, both our enzymatic and lipidomic analyses revealed surprisingly reduced cardiac TG levels in the hearts of our $\mathrm{Bscl}^{-/-}$mice (Figure 2). Different analytic approaches and/or distinct lines of $\mathrm{Bscl2}^{-/-}$mice likely explain these discrepancies. In the current study, comparison between lipidomes from wild-type and $B s c l 2^{-/}$hearts generated several interesting findings: among them, the dramatically reduced proportions of all 3 classes of glycerolipids as opposed to the increased proportions of phospholipids and sphingolipids in $\mathrm{Bscl2^{-/ }}$ hearts. Surprisingly, the levels of PA and DG, the important glycerolipid intermediates and phospholipid precursors, are reduced in $\mathrm{BsCl}^{-1}$ hearts. One potential mechanism is the surprising upregulation of ATGL-mediated accelerated intramyocellular TG turnover in $\mathrm{Bscl}^{-/-}$mice. The role of ATGL in cardiac TG turnover of $\mathrm{Bscl2}^{-/-}$mice is further supported by a slight recovery of cardiac TG content in hearts of $B s c l 2^{-1}$ mice with partial deletion of ATGL (Supplemental Table 2). Notably, mice with cardiac-specific overexpression of ATGL demonstrated reduced TG but not DG levels (52), suggesting additional pathways independent of ATGL may exist to exhaust cardiac glycerolipids in $\mathrm{Bscl}^{-/-}$hearts.

ATGL-mediated fat catabolism is known to provide ligands and activate PPAR $\alpha$ and PGC1 to regulate cardiac mitochondrial function (11). However, we failed to observe elevated mRNA levels of PPAR $\alpha$ and its FAO targets in $\mathrm{Bscl}^{-/-}$hearts (Figure 2). Lack of overt upregulation of PPAR $\alpha$ signaling in ATGL-elevated $\mathrm{Bscl2}^{-/-}$hearts may suggest an insufficient overexpression of ATGL in $\mathrm{Bscl} 2^{-/-}$hearts compared with about 20-fold upregulation in the hearts of animal models with cardiac overexpression of ATGL (52, 53). Despite that finding, cardiac ATGL upregulation may serve as an important trigger to induce a switch in cardiac substrate utilization to FAO by channeling more FAs to mitochondria in $\mathrm{Bscl2}^{-/-}$hearts. However, myocardial-specific ATGL overexpression has been shown to protect mice from both diabetes mellitus and diet-induced lipotoxic cardiomyopathy $(52,53)$, arguing against a direct role of cardiac ATGL overexpression in leading to cardiac dysfunction of $\mathrm{Bscl2}^{-/-}$mice.

Systemic insulin resistance is a primary factor that causes excessive FA flux leading to elevated FAO and mitochondrial function in obese and diabetic hearts (54). Different from obesity and type 2 diabetes mellitus, lipodystrophic $\mathrm{Bscl}^{-/-}$mice are hyperphagic but maintain a tendency toward lower circulating TG and NEFA levels in the ad libitum state $(23,25)$. Bscl2 $2^{--}$hearts demonstrated no change in TG-lipoprotein uptake (4). Although no difference in the expression of fatty acid transporters was found, it remains unknown whether lower circulating NEFA levels in severe insulin-resistant BSCL2 lipodystrophy could actually result from an even more avid uptake of NEFA by the heart. Meanwhile, excessive FA availability and oxidation is known to cause accumulation of long-chain FAs favorably incorporated into phospholipids (55). Bscl2 ${ }^{-1-}$ hearts demonstrate the signature pattern of aberrant phospholipid (especially cardiolipin) remodeling manifested as a proportional loss of the essential PUFA linoleic acid, paralleled by reciprocal increases in long-chain PUFAs, such as DHA, which is also commonly present in hearts of obese and diabetic animals $(56,57)$. Therefore, we reasoned that both ATGL upregulation and insulin resistance contributes to the excessive FAO and lipid remodeling in $\mathrm{BsCl}^{-/-}$hearts.

The reduced TG level and excessive FAO identified in $\mathrm{Bscl}^{-/-}$hearts are consistent with the role of BSCL2 in TG turnover and FAO, as reported in BSCL2-deleted adipocytes $(44,58)$. BSCL2 deletion in differentiating adipocytes causes unbridled cAMP/PKA signaling, which recruits more ATGL to lipid droplets to stimulate lipid catabolism (23). This was not the case in BSCL2-deleted hearts (Figure 2D). Instead, we identified enhanced ATGL protein stability in BSCL2-deleted cardiomyocytes and MEFs, suggesting a potential cell-autonomous role of BSCL2 in regulating ATGL turnover. NEFA has been shown to stabilize ATGL protein through inhibiting ubiquitination-mediated proteasome degradation in non-adipocyte cells (59). However, no significant upregulation of intracellular NEFA level was observed in the BSCL2-deleted heart (Supplemental Figure 2, A and B). Thus, how BSCL2 regulates ATGL protein stability and heart lipid catabolism needs to be further dissected with cardiomyocyte-restricted deletion of BSCL2 in vitro and in vivo.

Posttranslational lysine acetylation has emerged as a potentially important mechanism for controlling mitochondrial FAO and respiration (60). Specifically, protein acetylation of FAO enzymes is increased in hearts of high-fat diet-induced obese mice, which promotes enzyme activity, thereby increasing FAO rates in obesity (14). Like obesity, we also identified an increased overall mitochondrial protein acetylation associated with accelerated myocardial FAO rates and mitochondrial respiration in the hearts of lipodystrophic $\mathrm{Bscl2}^{-/-}$mice (Figure 3). Especially, increased acetylation of LCAD stimulates, rather than inhibits, its 
activity in $\mathrm{Bscl2}^{-/-}$hearts, similar to its regulation in obese hearts (14). Different from chronic high-fat diet feeding and diabetes mellitus $(14,46)$, we failed to identify a decrease in SIRT3 protein expression in Bscl2 $^{-1}$ hearts. Enhanced FAO may generate higher acetyl-CoA leading to nonenzymatic cardiac protein acetylation (61). Thus, it is difficult to conclude whether alteration of cardiac mitochondrial protein acetylation is secondary to changes in FAO. Nevertheless, reduced mitochondrial acetylation in $A^{h} B^{k}$ hearts was correlated to repressed LCAD activity and mitochondrial respiration, suggesting a significant role of mitochondrial acetylation in regulating cardiac FAO and mitochondrial bioenergetics in lipodystrophy.

It is well recognized that high FAO rates can decrease cardiac efficiency in insulin-resistant hearts (7, 62). Our results using heart homogenates consistently argues for a progressively enhanced FAO concomitant with a gradual inhibition of glucose oxidation, ultimately leading to metabolic cardiomyopathy in lipodystrophic $\mathrm{Bscl}^{-/-}$mice. Distinct from a previous study (4), we found no upregulation of O-GlcNAcylation of cardiac proteins in our $\mathrm{Bscl2}^{-/-}$mice (Supplemental Figure 3I), arguing against O-GlcNAcylation as a key element in pathological remodeling of hearts. Of note, altered substrate metabolism precedes cardiac contractile dysfunction in $B s c l 2^{-/}$mice, suggesting a causative role in the pathogenesis of cardiomyopathy related to BSCL2 lipodystrophy. Interestingly, increasing fat mass by partial deletion of ATGL in $B s c l 2^{-/-}$mice restored systemic and cardiac insulin sensitivity and normalized cardiac metabolic inflexibility (Figure 6). Considering that whole-body ATGL haploinsufficiency exerts no protection of hearts from diabetes-induced metabolic cardiomyopathy (52), the alleviation of cardiac dysfunction in $A^{h} B^{k}$ mice may be secondary to the improved systemic insulin resistance that is simply the result of restoration of adipose tissue mass. Further studies are necessary to demonstrate the causal link by using adipose tissue-specific deletion of ATGL in $\mathrm{Bscl}^{-1-}$ mice. Because of technical limitations, we did not directly assess FAO and glucose oxidation using an ex vivo-perfused working heart. Nevertheless, our study underscores a tight link between insulin resistance and metabolic substrate flexibility in leading to cardiomyopathy in lipodystrophy.

In conclusion, our results unveil a critical role of ATGL in mediating BSCL2 lipodystrophy and pinpoint aberrant lipid metabolism and mitochondrial protein acetylation as important contributors of metabolic cardiomyopathy in a unique diabetic model with lipodystrophy. Thus, partial inhibition of ATGL could not only specifically address the lack of functional adipose tissue in BSCL2 lipodystrophy, but also manage its associated insulin resistance and heart disease.

\section{Methods}

Mice. Global Bscl2-/- mice (backcrossed 5 times to the C57BL/6J background) were previously generated in the lab (23) and maintained under standard conditions with controlled 12-hour light/12-hour dark cycle and $21^{\circ} \mathrm{C} \pm 1{ }^{\circ} \mathrm{C}$ room temperature. Atgl/ Bscl2 double-KO mice were generated by breeding $\mathrm{Atgl}^{+/-}$mice (B6;129P2-Pnpla2 ${ }^{\text {tm1Rze} / J}$, Jackson stock 019003) with $\mathrm{Bscl2}^{+/-}$mice. Littermates from $\mathrm{Atgl}^{+/-} \mathrm{Bscl2}^{+/-}$mating were used for all experiments. Most experiments were performed in ad libitum-fed male mice and repeated in female mice. Mice were killed by cervical dislocation. Body compositions were measured using a Bruker small-animal NMR system (Bruker minispec LF90II).

Plasma biochemistry. Blood glucose levels were measured by OneTouch Ultra glucose meter. Insulin and leptin levels were measured using commercial ELISA kits (Millipore). Plasma IGF1 levels were measured using Mouse/Rat IGF-I Quantikine ELISA Kit (R\&D Systems). Glycerol and NEFA levels were determined using a free-glycerol reagent (Sigma-Aldrich) and WAKO NEFA analysis kit [NEFA-HR(2); Wako Pure Chemical Industries], respectively. Plasma TG and cholesterol levels were measured by colorimetrical analyses using a TG assay kit (Infinity Triglycerides kit, Thermo Fisher Scientific) and total cholesterol assay kit (Infinity Cholesterol Kit, Thermo Fisher Scientific) respectively.

Echocardiography. Transthoracic 2D and M-mode echocardiography analysis was used to assess heart function in conscious mice with a VisualSonics Vevo 2100 echocardiography machine equipped with a 30-MHz probe (VisualSonics).

Histology. Mouse adipose tissues were fixed, processed, and stained with H\&E. Whole hearts were fixed, embedded, and cut along the coronal plane to visualize the 4-chamber view.

Transmission electron microscopy. Left ventricle tissue was fixed in $4 \%$ paraformaldehyde, $2 \%$ glutaraldehyde in $0.1 \mathrm{M}$ sodium cacodylate ( $\mathrm{NaCac}$ ) buffer, $\mathrm{pH} 7.4$, postfixed in $2 \%$ osmium tetroxide in $\mathrm{NaCac}$, stained en bloc with $2 \%$ uranyl acetate, dehydrated with a graded ethanol series, and embedded in EponAraldite resin. Thin (70-nm) sections were cut with a diamond knife on a Leica EM UC6 ultramicrotome (Leica Microsystems, Inc.), collected on copper grids, and stained with uranyl acetate and lead citrate. 
Cells were observed in a JEM 1230 transmission electron microscope (JEOL USA Inc.) at $110 \mathrm{kV}$ and imaged with an UltraScan 4000 CCD camera and First Light Digital Camera Controller (Gatan Inc.).

Tissue and intracellular TG analyses, ORO, and lipidTOX staining. Tissues were homogenized in standard PBS buffer. Lipids were extracted and dissolved in chloroform. A small aliquot (5-30 $\mu \mathrm{L})$ was removed and dried for quantification. Cultured cells were directly lysed in 1\% Triton X-100 in PBS. The concentrations of TG were measured using a TG assay kit (Infinity Triglycerides kit). Data were normalized to tissue weights or total cellular protein levels as previously described (23). ORO and LipidTOX staining were performed as described previously (23).

RNA-seq and analysis. RNA was extracted by TRIzol (Thermo Fisher Scientific) from the hearts of 10-week-old $\mathrm{Bscl}^{+/+}$and $\mathrm{Bscl} 2^{-/-}$mice (female, 4-hour fast, $n=4$ with each pooled from 3 animals). Differential gene expression analysis was performed using RNA-seq at the Genome Technology Access Center at Washington University. The library was prepared using a Ribo-Zero gold rRNA removal kit and paired-end sequencing of 100-base length was performed on a HiSeq 3000 system (Illumina). RNA-seq reads were then aligned to the Ensembl release 76 top-level assembly with STAR version 2.0.4b. For each contrast extracted with Limma, global perturbations in known KEGG pathways were detected using the R/Bioconductor package GAGE. The R/Bioconductor package heatmap3 and Pathview was used to display heatmaps or annotated KEGG graphs across groups of samples for each KEGG pathway with a Benjamini-Hochberg false-discovery rate adjusted $P$ value less than or equal to 0.05 . All data have been submitted to the NCBI's Sequence Read Archive (SRA, accession PRJNA542823).

Lipidomic analysis by high-resolution/accurate mass spectrometry and tandem mass spectrometry. Frozen ventricles were homogenized in methanol/chloroform/water (2:1:0.74) as previously described (63). Prior to lipid extraction, each tissue homogenate was spiked with synthetic PC (14:0/14:0), PE (14:0/14:0), and PS (14:0/14:0) obtained from Avanti Polar Lipids at $1 \mathrm{nmol} / \mathrm{mg}$ tissue as internal standards for relative lipid quantitation. Dried lipid extracts were washed 3 times with $10 \mathrm{mM}$ ammonium bicarbonate, dried under vacuum, and resuspended in isopropanol/methanol/chloroform (4:2:1, v/v/v) using 100 $\mu \mathrm{L} / \mathrm{mg}$ tissue extracted. For each analysis, $40 \mu \mathrm{L}$ of lipid extract was transferred to an Eppendorf twintec 96-well plate (Sigma-Aldrich), and evaporated under nitrogen. The dried lipid film was then resuspended in isopropanol/methanol/chloroform (4:2:1 v/v/v) containing $20 \mathrm{mM}$ ammonium formate and sealed with Teflon Ultra-Thin Sealing Tape (Analytical Sales and Services). Untargeted lipidomic analysis was performed by directly infusing samples to the mass spectrometer by nanoelectrospray ionization (nESI) using an Advion Triversa Nanomate nESI source (Advion) with a spray voltage of $1.4 \mathrm{kV}$ and a gas pressure of 0.3 psi. High-resolution MS and MS/MS spectra were acquired in both positive and negative ionization modes using the FT analyzer operating at 100,000 mass resolving power. Ion mapping higher-energy collision-induced dissociation (HCD-MS/MS) product ion spectra were acquired to confirm lipid headgroups and acyl chain compositions. Lipids were identified using the Lipid Mass Spectrum Analysis (LIMSA) v.1.0 software linear fit algorithm, in conjunction with a user-defined database of hypothetical lipid compounds for automated peak finding and correction of ${ }^{13} \mathrm{C}$ isotope effects. Relative quantification of abundance between samples was performed by normalization of target lipid ion peak areas to the PC (14:0/14:0) internal standard as previously described (64).

Insulin tolerance tests and cardiac insulin signaling. Insulin tolerance tests were performed in mice fasted 6 hours and then injected i.p. with human insulin (Humulin, Novo Nordisk) at $0.75 \mathrm{U} / \mathrm{kg}$. Blood glucose levels were measured by OneTouch Ultra glucose meter before and at 15, 30, 60, and 120 minutes after injection. To detect cardiac insulin signaling, mice were fasted for 4 hours followed by i.p. injection of Humulin (1.0 U/kg BW). Exactly 15 minutes later, ventricles were excised and immediately snap frozen in liquid nitrogen for Western blot analyses of insulin-mediated signaling.

FA and glucose oxidation assays. FAO and glucose oxidation reaction assays with left-ventricle homogenates were prepared and carried out as detailed previously (65). Briefly, approximately 25-mg pieces of freshly isolated ventricle tissues were homogenized in STE buffer (250 mM sucrose, $10 \mathrm{mM}$ Tris [pH 7.5], and $1 \mathrm{mM}$ EDTA) using a glass dounce homogenizer (20 loose and 20 tight strokes). Homogenates were incubated with $300 \mu \mathrm{M}$ cold BSA-conjugated palmitate and $1 \mu \mathrm{Ci} / \mathrm{mL}\left[1-{ }^{14} \mathrm{C}\right]$ palmitic acid substrate for FAO or $200 \mu \mathrm{M}$ cold glucose and $0.1 \mu \mathrm{Ci} \mathrm{D}-\left[{ }^{14} \mathrm{C}(\mathrm{U})\right]$-glucose/reaction for glucose oxidation. The released $\left[{ }^{14} \mathrm{C}\right] \mathrm{CO}_{2}$ was captured by hydroamine-soaked filter paper and measured by scintillation counting, while ASMs were analyzed by centrifugation and counting of ${ }^{14} \mathrm{C}$ radioactivity in the supernatant. Data were normalized to the total protein content for LV homogenates. 
$T G$ hydrolase activity assay. Cardiac TG hydrolase activity was measured as described previously (66). Briefly, fresh ventricle tissue samples (20-30 mg) were homogenized in $500 \mu \mathrm{L}$ buffer A ( $0.25 \mathrm{M}$ sucrose, $1 \mathrm{mM}$ EDTA, and protease inhibitor cocktail) using a Bullet Blender homogenizer. Homogenates were first centrifuged at $1,000 \mathrm{~g}$ for 5 minutes, then at $20,000 \mathrm{~g}$ for 3 minutes at $4^{\circ} \mathrm{C}$. The clear lysates were transferred into new microtube without floating lipid or pellet for protein assay. Reactions performed by incubating samples in a total volume of $100 \mu \mathrm{L}$ buffer A with $100 \mu \mathrm{L}$ TG substrate in a water bath at $37^{\circ} \mathrm{C}$ for 60 minutes. Reactions were terminated by adding $3.25 \mathrm{~mL}$ of methanol/chloroform/heptane (10:9:7) and $1 \mathrm{~mL}$ of $0.1 \mathrm{M}$ potassium carbonate and $0.1 \mathrm{M}$ boric acid $(\mathrm{pH} 10.5)$ followed by centrifugation $(800 \mathrm{~g}$, 15 minutes). After centrifugation, the radioactivity in $1 \mathrm{~mL}$ of the upper phase was determined by liquid scintillation counting. Counts from control incubations were subtracted and the rate of FA hydrolysis was calculated using ${ }^{3} \mathrm{H}$ radiolabeling of triolein substrate. TG substrate was prepared by emulsifying $330 \mu \mathrm{M}$ triolein $(40,000 \mathrm{cpm} / \mathrm{nmol})$ and $45 \mu \mathrm{M}$ PC/PI (3:1) in $100 \mathrm{mM}$ potassium phosphate buffer (pH 7.0) by sonication and adjusted to $5 \%$ essentially FA-free BSA.

Mitochondrial DNA content and bioenergetics. Total DNA was extracted from ventricles and mitochondrial DNA content was measured by qPCR as described previously (58). For Seahorse bioenergetics analysis, fresh ventricles were minced in ice-cold fiber relaxation buffer and homogenized in a glass dounce homogenizer followed by mitochondria isolation and immediate analysis in an XF24 Analyzer (Seahorse Bioscience) as previously described (67). Briefly, mitochondrial protein yield was determined by Bradford assay and $3 \mu \mathrm{g}$ mitochondria was seeded per well by centrifugation. Basal OCR was measured in the presence of $10 \mathrm{mM}$ succinate and $2 \mu \mathrm{M}$ rotenone. Electron flow assays were performed by measuring basal OCR in the presence of $10 \mathrm{mM}$ pyruvate (complex I substrate), $2 \mathrm{mM}$ malate, and $4 \mu \mathrm{M}$ FCCP, and after sequential addition of $2 \mu \mathrm{M}$ rotenone (complex I inhibitor), $10 \mathrm{mM}$ succinate (complex II substrate), $4 \mu \mathrm{M}$ antimycin A (complex III inhibitor), and $1 \mathrm{mM} N, N, N^{\prime}, N^{\prime}$-tetramethyl-p-phenylenediamine (TMPD) containing 10 $\mathrm{mM}$ ascorbate (complex IV substrate). Complex III respiration corresponds to the antimycin A-sensitive respiration. OCR was normalized per microgram of mitochondrial protein.

Assessment of LCAD activity. LCAD activity was assayed based on the method described by Lehman et al. (68). In brief, ventricles were homogenized in buffer containing $250 \mathrm{mM}$ sucrose, $20 \mathrm{mM}$ Tris, $40 \mathrm{mM} \mathrm{KCl}$, and 2 mM EGTA, pH 7.4. Total heart lysate $(20 \mu \mathrm{g})$ was added to potassium buffer containing $200 \mu \mathrm{M}$ ferrocenium hexafluorophosphate, $500 \mu \mathrm{M} N$-ethylmaleimide, and $0.1 \mathrm{mM}$ EDTA at pH 7.2. Palmitoyl-CoA (50 $\mu \mathrm{M}$ ) was added to initiate the reaction and the absorbance at 300-nm wavelength was followed for 5 minutes using a BioTek spectrophotometer kinetic plate reader. Data were normalized to protein.

Isolation and culture of adult cardiomyocytes. The isolation of adult mouse cardiomyocytes was carried out based on established procedures (69). The cardiomyocytes were suspended in plating media and plated onto laminin-precoated $(5 \mu \mathrm{g} / \mathrm{mL})$ tissue culture plates. One hour after plating, cells were changed to culture media and treated with bortezomib, cycloheximide, or vehicle control (DMSO).

Isolation and differentiation of MEFs and SVCs. MEFs were isolated from 12.5- to 14.5-day-old embryos and SVCs were isolated from 6-week-old sWAT as previously described (23). MEFs or SVCs were plated at the same density and maintained to 2 days after confluence (D0). Differentiation was induced by using conventional differentiation cocktails DMI (dexamethasone, IBMX, and insulin) for 2 days followed by regular media (DMEM high glucose plus 10\% FBS and pen/strep) in the presence of $100 \mathrm{nM}$ insulin alone for another 2 days. In MEF experiments, the ATGL-specific inhibitor Atglistatin (SML1075-25MG, Sigma-Aldrich) was added to regular culture media starting with 4 days after differentiation and constantly maintained in the later culture with vehicle included in the control cells.

Measurement of reactive oxygen species. Fluorescence of reaction oxygen species (ROS) were detected by incubating frozen sections with $5 \mu \mathrm{M}$ DCFDA (Invitrogen) for 30 minutes. MDA, an indicator of lipid peroxidation as a part of thiobarbituric acid-reactive substances (TBARS), was assayed using a TBARS Assay Kit (Cayman Chemical) as instructed.

$R N A$ isolation and real-time quantitative PCR. Total RNA was extracted with TRIzol and reverse transcribed using MLV-V reverse transcriptase using random primers (Invitrogen). Real-time quantitative RT-PCR was performed on the Stratagene MX3005 system. Data were normalized to 2 housekeeping genes ( $\beta$-actin and 36B4) based on the Genorm algorithm (medgen.ugent.be/genorm/) and expressed as fold changes relative to wild-type mice. All tissue gene expression studies were performed in nonfasted mice.

Muscle fractionation and membrane localization of PKC. Left ventricles ( $20 \mathrm{mg})$ were homogenized in a bullet blender in Buffer A (20 mM Tris- $\mathrm{HCl}$ pH 7.5, 1 mM EDTA, 0.25 mM EGTA, and 0.25 M sucrose) with 
freshly added protease and phosphatase inhibitor cocktails (Sigma-Aldrich). Homogenized samples were first spun at $400 \mathrm{~g}$ for 15 minutes. The supernatant was then centrifuged at 105,000 $\mathrm{g}$ for 45 minutes. The pellet (membrane) fraction was resuspended in $50 \mu \mathrm{L}$ Buffer A and then mixed with $100 \mu \mathrm{L}$ Buffer B (Buffer A without sucrose but 2\% Triton X-100). Proteins were solubilized, quantified, and used for Western blot.

Immunoblotting. Tissues were lysed in lysis buffer containing $25 \mathrm{mM}$ Tris- $\mathrm{HCl}(\mathrm{pH} 7.4), 150 \mathrm{mM} \mathrm{NaCl}$, 2 mM EDTA, 1\% Triton X-100, and 10\% glycerol with freshly added protease and phosphatase inhibitor cocktail (Sigma-Aldrich). The protein concentration was determined by Bradford protein assay (Bio-Rad). Equivalent amounts of homogenate protein were resolved by SDS-polyacrylamide gels and transferred to nitrocellulose membranes. The blots were probed with specific antibodies, developed using the ECL chemiluminescence system, and imaged by AMERSHAM Imager 600 (GE Healthcare) followed by densitometry analysis using ImageQuantTL (GE Healthcare).

The following antibodies were used: rabbit antibodies against phospho-AKT (Ser473) (catalog 4060), AKT (catalog 9272), phospho-PKA substrate (catalog 9624), HSL (catalog 4107), IGF1R

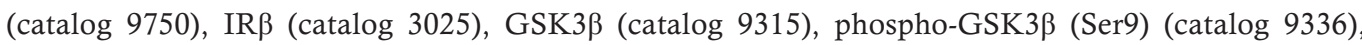
phospho-p70 S6 kinase (Thr389) (catalog 9234), p70 S6 kinase (catalog 2708), SIRT3 (catalog 5490), acetylated lysine (catalog 9441), PPAR $\gamma$ (2435) (all from Cell Signaling Technology); CD36 (cata-

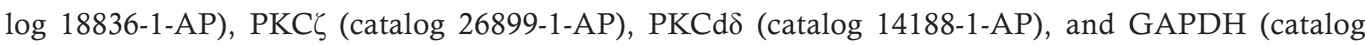
60004-1-IG) (all from Proteintech); ATGL (catalog 10006409; Cayman Chemical); CATA (catalog GTX110704) and SOD2 (catalog GTX116093) (both from Genetex); Total OXPHOS Rodent WB Antibody Cocktail (catalog ab110413, Abcam); $\beta$-actin (catalog MAB1501, MillporeSigma); phospho-IR/ IGF1R (Tyr1158/Tyr1162/Tyr1163) (catalog 07-841, MilliporeSigma); PKC $\varepsilon$ (catalog sc-1681, Santa Cruz Biotechnology); PLIN1 (catalog GP29, Progen Biotechnik GmbH). PLIN2 is an antibody that was produced in-house as previously reported (44).

Statistics. Quantitative data are presented as means \pm SEM. Animal experiments were performed with at least 3 independent cohorts. Differences between groups were examined for statistical significance with an unpaired $t$ test, 1-way ANOVA corrected with Dunnett's multiple-comparisons test, 2-way ANOVA followed by Tukey's or Dunnett's post hoc tests, or multiple $t$ tests after correction using the Holm-Sidak method. All these analyses were performed using GraphPad Prism version 8. A $P$ value of less than 0.05 was considered statistically significant.

Study approval. All animal experiments were done according to the NIH Guide for the Care and Use of Laboratory Animals (National Academies Press, 2011) and approved by the IACUC at Augusta University.

\section{Author contributions}

$\mathrm{HZ}$ and WC designed the study, performed the experiments, and wrote the manuscript. XL, YY, and JL performed the experiments. TL performed the lipidomic analysis. NLW and HS discussed the projects and revised the manuscript. All authors agree to the content of the final manuscript.

\section{Acknowledgments}

We acknowledge Yisang Yoon and Hakjoo Lee from the Department of Physiology at Augusta University for technical help with cardiac mitochondria isolation and measurement of oxygen consumption. We thank the Electron Microscopy and Histology Core at Augusta University for technical assistance and electron microscope imaging. We also thank Hongyan $\mathrm{Xu}$ from the Department of Population Health Sciences for his help with statistical analyses. This work was supported by the National Heart, Lung, and Blood Institute at the NIH (1R01HL132182-01 to WC) and (R01 HL124248 to HS) as well as the American Heart Association Grant-in-aid (16GRNT30680004 to WC) and the American Heart Association Career Development Award (18CDA34080244 to HZ).

Address correspondence to: Weiqin Chen, 1120 15th Street, CA3094, Department of Physiology, Medical College of Georgia at Augusta University, Augusta, Georgia 30912 USA. Phone: 706.721.8706; Email: wechen@augusta.edu.

XL's present address is: College of Veterinary Medicine, Hunan Agricultural University, Changsha, Hunan Province, China. 
1. Kolwicz SC, Tian R. Glucose metabolism and cardiac hypertrophy. Cardiovasc Res. 2011;90(2):194-201.

2. McGavock JM, et al. Cardiac steatosis in diabetes mellitus: a 1H-magnetic resonance spectroscopy study. Circulation. 2007;116(10):1170-1175.

3. Rijzewijk LJ, et al. Myocardial steatosis is an independent predictor of diastolic dysfunction in type 2 diabetes mellitus. $J$ Am Coll Cardiol. 2008;52(22):1793-1799.

4. Joubert M, et al. The sodium-glucose cotransporter 2 inhibitor dapagliflozin prevents cardiomyopathy in a diabetic lipodystrophic mouse model. Diabetes. 2017;66(4):1030-1040.

5. Liu S, et al. Development of hypertrophic cardiomyopathy in perilipin-1 null mice with adipose tissue dysfunction. Cardiovasc Res. 2015;105(1):20-30.

6. van der Vusse GJ, Glatz JF, Stam HC, Reneman RS. Fatty acid homeostasis in the normoxic and ischemic heart. Physiol Rev. 1992;72(4):881-940.

7. Buchanan J, et al. Reduced cardiac efficiency and altered substrate metabolism precedes the onset of hyperglycemia and contractile dysfunction in two mouse models of insulin resistance and obesity. Endocrinology. 2005;146(12):5341-5349.

8. Liu L, et al. DGAT1 deficiency decreases PPAR expression and does not lead to lipotoxicity in cardiac and skeletal muscle. J Lipid Res. 2011;52(4):732-744.

9. Son NH, et al. PPAR $\gamma$-induced cardiolipotoxicity in mice is ameliorated by PPAR $\alpha$ deficiency despite increases in fatty acid oxidation. J Clin Invest. 2010;120(10):3443-3454.

10. Banke NH, et al. Preferential oxidation of triacylglyceride-derived fatty acids in heart is augmented by the nuclear receptor PPARalpha. Circ Res. 2010;107(2):233-241.

11. Haemmerle G, et al. ATGL-mediated fat catabolism regulates cardiac mitochondrial function via PPAR- $\alpha$ and PGC-1. Nat Med. 2011;17(9):1076-1085

12. O'Donnell JM, Fields AD, Sorokina N, Lewandowski ED. The absence of endogenous lipid oxidation in early stage heart failure exposes limits in lipid storage and turnover. J Mol Cell Cardiol. 2008;44(2):315-322.

13. Anderson KA, Hirschey MD. Mitochondrial protein acetylation regulates metabolism. Essays Biochem. 2012;52:23-35.

14. Alrob OA, et al. Obesity-induced lysine acetylation increases cardiac fatty acid oxidation and impairs insulin signalling. Cardiovasc Res. 2014;103(4):485-497.

15. Romanick SS, et al. Obesity-mediated regulation of cardiac protein acetylation: parallel analysis of total and acetylated proteins via TMT-tagged mass spectrometry. Biosci Rep. 2018;38(5):BSR20180721.

16. Berardinelli W. An undiagnosed endocrinometabolic syndrome: report of 2 cases. J Clin Endocrinol Metab. 1954;14(2):193-204

17. Seip M, Trygstad O. Generalized lipodystrophy, congenital and acquired (lipoatrophy). Acta Paediatr Suppl. 1996;413:2-28.

18. Bjørnstad PG, Foerster A, Ihlen H. Cardiac findings in generalized lipodystrophy. Acta Paediatr Suppl. 1996;413:39-43.

19. Bhayana S, Siu VM, Joubert GI, Clarson CL, Cao H, Hegele RA. Cardiomyopathy in congenital complete lipodystrophy. Clin Genet. 2002;61(4):283-287.

20. Friguls B, Coroleu W, del Alcazar R, Hilbert P, Van Maldergem L, Pintos-Morell G. Severe cardiac phenotype of Berardinelli-Seip congenital lipodystrophy in an infant with homozygous E189X BSCL2 mutation. Eur J Med Genet. 2009;52(1):14-16.

21. Lupsa BC, Sachdev V, Lungu AO, Rosing DR, Gorden P. Cardiomyopathy in congenital and acquired generalized lipodystrophy: a clinical assessment. Medicine (Baltimore). 2010;89(4):245-250.

22. Magré J, et al. Identification of the gene altered in Berardinelli-Seip congenital lipodystrophy on chromosome 11q13. Nat Genet. 2001;28(4):365-370

23. Chen W, et al. Berardinelli-seip congenital lipodystrophy 2 /seipin is a cell-autonomous regulator of lipolysis essential for adipocyte differentiation. Mol Cell Biol. 2012;32(6):1099-1111.

24. Cui X, et al. Seipin ablation in mice results in severe generalized lipodystrophy. Hum Mol Genet. 2011;20(15):3022-3030.

25. Prieur X, et al. Thiazolidinediones partially reverse the metabolic disturbances observed in Bscl2/seipin-deficient mice. Diabetologia. 2013;56(8):1813-1825

26. Chen W, Yechoor VK, Chang BH, Li MV, March KL, Chan L. The human lipodystrophy gene product Berardinelli-Seip congenital lipodystrophy 2/seipin plays a key role in adipocyte differentiation. Endocrinology. 2009;150(10):4552-4561.

27. Fei W, et al. Fld1p, a functional homologue of human seipin, regulates the size of lipid droplets in yeast. J Cell Biol. 2008;180(3):473-482.

28. Romanauska A, Köhler A. The inner nuclear membrane is a metabolically active territory that generates nuclear lipid droplets Cell. 2018;174(3):700-715.e18.

29. Szymanski KM, et al. The lipodystrophy protein seipin is found at endoplasmic reticulum lipid droplet junctions and is important for droplet morphology. Proc Natl Acad Sci U S A. 2007;104(52):20890-20895.

30. Wang S, Idrissi FZ, Hermansson M, Grippa A, Ejsing CS, Carvalho P. Seipin and the membrane-shaping protein Pex30 cooperate in organelle budding from the endoplasmic reticulum. Nat Commun. 2018;9(1):2939.

31. Bi J, et al. Seipin promotes adipose tissue fat storage through the ER Ca ${ }^{2+}$-ATPase SERCA. Cell Metab. 2014;19(5):861-871.

32. Ding L, et al. Seipin regulates lipid homeostasis by ensuring calcium-dependent mitochondrial metabolism. EMBO J. 2018;37(17):e97572.

33. Wang H, et al. Seipin is required for converting nascent to mature lipid droplets. Elife. 2016;5:e16582.

34. Sui X, et al. Cryo-electron microscopy structure of the lipid droplet-formation protein seipin. J Cell Biol. 2018;217(12):4080-4091.

35. Yan R, et al. Human SEIPIN binds anionic phospholipids. Dev Cell. 2018;47(2):248-256.e4.

36. Talukder MM, Sim MF, O'Rahilly S, Edwardson JM, Rochford JJ. Seipin oligomers can interact directly with AGPAT2 and lipin 1, physically scaffolding critical regulators of adipogenesis. Mol Metab. 2015;4(3):199-209.

37. Sim MF, et al. The human lipodystrophy protein seipin is an ER membrane adaptor for the adipogenic PA phosphatase lipin 1. Mol Metab. 2012;2(1):38-46.

38. Pagac M, et al. SEIPIN regulates lipid droplet expansion and adipocyte development by modulating the activity of glycerol-3-phosphate acyltransferase. Cell Rep. 2016;17(6):1546-1559.

39. Castro IG, Eisenberg-Bord M, Persiani E, Rochford JJ, Schuldiner M, Bohnert M. Promethin is a conserved seipin partner protein. Cells. 2019;8(3):E268. 
40. Maillet M, van Berlo JH, Molkentin JD. Molecular basis of physiological heart growth: fundamental concepts and new players. Nat Rev Mol Cell Biol. 2013;14(1):38-48.

41. Lanktree MB, Johansen CT, Joy TR, Hegele RA. A translational view of the genetics of lipodystrophy and ectopic fat deposition. Prog Mol Biol Transl Sci. 2010;94:159-196.

42. Athenstaedt K, Daum G. Phosphatidic acid, a key intermediate in lipid metabolism. Eur J Biochem. 1999;266(1):1-16.

43. Schlame M, Brody S, Hostetler KY. Mitochondrial cardiolipin in diverse eukaryotes. Comparison of biosynthetic reactions and molecular acyl species. Eur J Biochem. 1993;212(3):727-735.

44. Zhou H, et al. Berardinelli-Seip congenital lipodystrophy 2 regulates adipocyte lipolysis, browning, and energy balance in adult animals. J Lipid Res. 2015;56(10):1912-1925.

45. Dai Z, et al. Dual regulation of adipose triglyceride lipase by pigment epithelium-derived factor: a novel mechanistic insight into progressive obesity. Mol Cell Endocrinol. 2013;377(1-2):123-134.

46. Hirschey MD, et al. SIRT3 deficiency and mitochondrial protein hyperacetylation accelerate the development of the metabolic syndrome. Mol Cell. 2011;44(2):177-190.

47. Schweiger M, et al. Pharmacological inhibition of adipose triglyceride lipase corrects high-fat diet-induced insulin resistance and hepatosteatosis in mice. Nat Commun. 2017;8:14859.

48. Haemmerle G, et al. Defective lipolysis and altered energy metabolism in mice lacking adipose triglyceride lipase. Science. 2006;312(5774):734-737.

49. Gavrilova O, et al. Surgical implantation of adipose tissue reverses diabetes in lipoatrophic mice. J Clin Invest. 2000;105(3):271-278.

50. Rheuban KS, Blizzard RM, Parker MA, Carter T, Wilson T, Gutgesell HP. Hypertrophic cardiomyopathy in total lipodystrophy. J Pediatr. 1986;109(2):301-302.

51. Nelson MD, Victor RG, Szczepaniak EW, Simha V, Garg A, Szczepaniak LS. Cardiac steatosis and left ventricular hypertrophy in patients with generalized lipodystrophy as determined by magnetic resonance spectroscopy and imaging. Am J Cardiol. 2013;112(7):1019-1024.

52. Pulinilkunnil T, et al. Myocardial adipose triglyceride lipase overexpression protects diabetic mice from the development of lipotoxic cardiomyopathy. Diabetes. 2013;62(5):1464-1477.

53. Pulinilkunnil T, Kienesberger PC, Nagendran J, Sharma N, Young ME, Dyck JR. Cardiac-specific adipose triglyceride lipase overexpression protects from cardiac steatosis and dilated cardiomyopathy following diet-induced obesity. Int J Obes (Lond). 2014;38(2):205-215.

54. Witteles RM, Fowler MB. Insulin-resistant cardiomyopathy clinical evidence, mechanisms, and treatment options. J Am Coll Cardiol. 2008;51(2):93-102.

55. Goldberg IJ, Trent CM, Schulze PC. Lipid metabolism and toxicity in the heart. Cell Metab. 2012;15(6):805-812.

56. Han X, Yang J, Yang K, Zhao Z, Abendschein DR, Gross RW. Alterations in myocardial cardiolipin content and composition occur at the very earliest stages of diabetes: a shotgun lipidomics study. Biochemistry. 2007;46(21):6417-6428.

57. Watkins SM, Reifsnyder PR, Pan HJ, German JB, Leiter EH. Lipid metabolome-wide effects of the PPAR $\gamma$ agonist rosiglitazone. J Lipid Res. 2002;43(11):1809-1817.

58. Zhou H, Black SM, Benson TW, Weintraub NL, Chen W. Berardinelli-seip congenital lipodystrophy 2/Seipin is not required for brown adipogenesis but regulates brown adipose tissue development and function. Mol Cell Biol. 2016;36(15):2027-2038 .

59. Olzmann JA, Richter CM, Kopito RR. Spatial regulation of UBXD8 and p97/VCP controls ATGL-mediated lipid droplet turnover. Proc Natl Acad Sci U S A. 2013;110(4):1345-1350.

60. Hirschey MD, et al. SIRT3 regulates mitochondrial fatty-acid oxidation by reversible enzyme deacetylation. Nature. 2010;464(7285):121-125.

61. Parodi-Rullán RM, Chapa-Dubocq XR, Javadov S. Acetylation of mitochondrial proteins in the heart: the role of SIRT3. Front Physiol. 2018;9:1094

62. Mazumder PK, et al. Impaired cardiac efficiency and increased fatty acid oxidation in insulin-resistant ob/ob mouse hearts. Diabetes. 2004;53(9):2366-2374.

63. Lydic TA, Busik JV, Reid GE. A monophasic extraction strategy for the simultaneous lipidome analysis of polar and nonpolar retina lipids. J Lipid Res. 2014;55(8):1797-1809.

64. Lydic TA, Townsend S, Adda CG, Collins C, Mathivanan S, Reid GE. Rapid and comprehensive 'shotgun' lipidome profiling of colorectal cancer cell derived exosomes. Methods. 2015;87:83-95.

65. Xu W, Zhou H, Xuan H, Saha P, Wang G, Chen W. Novel metabolic disorders in skeletal muscle of Lipodystrophic Bsc12/ Seipin deficient mice. Mol Cell Endocrinol. 2019;482:1-10.

66. Schweiger M, et al. G0/G1 switch gene-2 regulates human adipocyte lipolysis by affecting activity and localization of adipose triglyceride lipase. J Lipid Res. 2012;53(11):2307-2317.

67. Vergnes L, et al. Adipocyte browning and higher mitochondrial function in periadrenal but not SC fat in pheochromocytoma. J Clin Endocrinol Metab. 2016;101(11):4440-4448.

68. Lehman TC, Hale DE, Bhala A, Thorpe C. An acyl-coenzyme A dehydrogenase assay utilizing the ferricenium ion. Anal Biochem. 1990;186(2):280-284.

69. Ackers-Johnson M, Li PY, Holmes AP, O’Brien SM, Pavlovic D, Foo RS. A simplified, Langendorff-free method for concomitant isolation of viable cardiac myocytes and nonmyocytes from the adult mouse heart. Circ Res. 2016;119(8):909-920. 